\title{
Overbarrier reflection in quantum mechanics with multiple degrees of freedom
}

\author{
D. G. Levkov, ${ }^{1, *}$ A. G. Panin, ${ }^{1,2, \dagger}$ and S. M. Sibiryakov ${ }^{1,3, *}$ \\ ${ }^{1}$ Institute for Nuclear Research of the Russian Academy of Sciences, 60th October Anniversary Prospect 7a, Moscow 117312, Russia \\ ${ }^{2}$ Moscow Institute of Physics and Technology, Institutskii per. 9, Dolgoprudny 141700, Moscow Region, Russia \\ ${ }^{3}$ Theory Group, Physics Department, CERN, CH-1211 Geneva 23, Switzerland
}

(Received 10 April 2007; published 21 September 2007)

\begin{abstract}
We present an analytic example of a two-dimensional quantum-mechanical system, where the exponential suppression of the probability of overbarrier reflection changes nonmonotonically with energy. The suppression is minimal at certain "optimal" energies where reflection occurs with exponentially larger probability than at other energies.
\end{abstract}

DOI: 10.1103/PhysRevA.76.032114

PACS number(s): 03.65.Sq, 03.65.Xp

\section{INTRODUCTION}

Tunneling and overbarrier reflection are the characteristic nonperturbative phenomena in quantum mechanics. They typically occur with exponentially small probabilities,

$$
\mathcal{P} \propto e^{-F / \hbar},
$$

where $F$ is the suppression exponent; still, the above phenomena are indispensable in understanding a wide variety of physical situations, from the generation of baryon number asymmetry in the early Universe [1] to chemical reactions [2] and atom ionization processes [3].

During the last decades extensive investigations of tunneling processes in systems with many degrees of freedom have been performed $[2,4-13]$. These studies revealed a rich variety of features of multidimensional tunneling which are in striking contrast to the properties of one-dimensional tunneling and overbarrier reflection. In particular, the following phenomenon has been observed: the probability of tunneling may depend nonmonotonically on the total energy of the system and exhibit resonancelike peaks. One can envisage three physically different mechanisms of this phenomenon. The first mechanism, present already in the one-dimensional case, is tunneling via creation of a metastable state. In this case the tunneling probability at the maximum of the resonance is exponentially higher than at other energies. On the other hand, the resonance width $\Delta E$ is exponentially suppressed; so, after averaging with an energy distribution of a finite width the effect of the resonance is washed out in the semiclassical limit $\hbar \rightarrow 0$. The second possible mechanism of nonmonotonic behavior of $\mathcal{P}(E)$ is quantum interference [7,13] (see also [14]). In this case the peak value of the tunneling probability is only by a factor of order one higher than the average value, while the width of the resonance scales as $\Delta E \propto \hbar$. Again, the resonances become indiscernible in the semiclassical limit. In both these cases the resonances can be attributed to the subleading semiclassical corrections, i.e., nonmonotonic behavior of the preexponential factor omitted in Eq. (1). The third possibility is that the suppres-

\footnotetext{
*levkov@ms2.inr.ac.ru

†panin@ms2.inr.ac.ru

†Sergey.Sibiryakov@cern.ch; sibir@ms2.inr.ac.ru
}

sion exponent $F(E)$ is nonmonotonic. In this case the existence of the "resonances" is the leading semiclassical effect: the optimal tunneling probability at the maximum of the resonance is exponentially higher than the probability at other energies. At the same time the resonance width scales as $^{1} \Delta E \propto \sqrt{\hbar}$. This last possibility of "optimal tunneling" is definitely of interest; yet, it did not receive much attention in literature. We are aware of only a few works mentioning nonmonotonic dependence of the suppression exponent on energy [14-16]. It is worthwhile studying this phenomenon in detail; this can provide insight into the dynamics of multidimensional tunneling.

In this paper we consider the process of overbarrier reflection in a simple model with two degrees of freedom. Our setup is interesting in two respects. First, the model under study is essentially nonlinear and the variables cannot be separated; still, overbarrier reflections in this model can be described analytically within the semiclassical framework. Thus, this model can serve as an analytic laboratory for the study of multidimensional tunneling. Second, the suppression exponent $F$ of the reflection process behaves nonmonotonically as the total energy $E$ changes. We demonstrate that the function $F(E)$ possesses a number of local minima $E$ $=E_{o}$, where reflection is optimal. We stress that the process we study is exponentially preferable at "optimal" energies as compared to other energies.

Our model describes the motion of a quantum particle in the two-dimensional harmonic waveguide (see Refs. $[8,10,14]$ for similar models). The Hamiltonian is

$$
H=\frac{p_{x}^{2}}{2 m}+\frac{p_{y}^{2}}{2 m}+\frac{m \omega^{2}}{2} w^{2}(x, y),
$$

where $x, y$ are the Cartesian coordinates and $m$ is the mass of the particle. The function $U=m \omega^{2} w^{2} / 2$ represents the waveguide potential in two dimensions: a particle with small energy is bound to move along the line $w(x, y) \approx 0$. We do not

\footnotetext{
${ }^{1}$ This follows from the representation

$$
\mathcal{P}(E) \propto \exp \left(-\frac{F\left(E_{o}\right)}{\hbar}-\frac{F^{\prime \prime}\left(E_{o}\right)\left(E-E_{o}\right)^{2}}{2 \hbar}\right)
$$
}

of the tunneling probability in the vicinity of the maximum. 

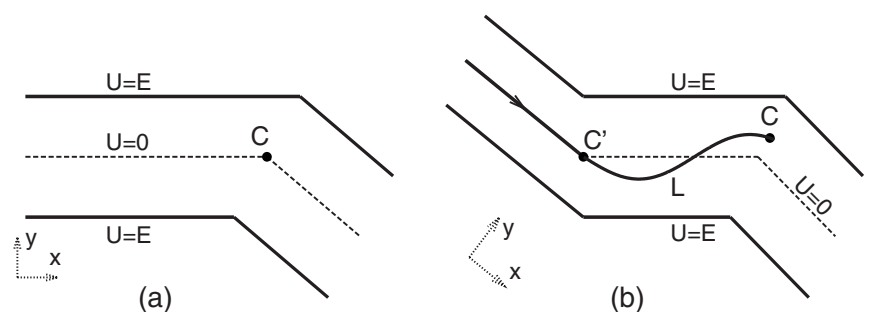

FIG. 1. The equipotential contour $U=E$ for the waveguides with (a) one and (b) two sharp turns. An example of classical trajectory is shown in case(b).

introduce a potential barrier across the waveguide and consider the case when the line $w=0$ stretches all the way from $x \rightarrow-\infty$ to $x \rightarrow+\infty$. We also assume that the function $w(x, y)$ is linear in the initial asymptotic region,

$$
w(x, y) \rightarrow y \quad \text { as } x \rightarrow-\infty
$$

In the present paper we consider two particular cases of the function $w(x, y)$ describing waveguides with one and two sharp turns, ${ }^{2}$ see Fig. 1.

The motion of the particle at $x \rightarrow-\infty$ is a superposition of free translatory motion in $x$ direction and oscillations of frequency $\omega$ along $y$ coordinate; the state of such a particle is fully characterized by two quantum numbers, the total energy $E$ and $y$-oscillator excitation number $N$. The particle sent into the waveguide from the asymptotic region $x \rightarrow-\infty$ with given $E, N$ may either continue to move towards $x \rightarrow+\infty$, or reflect back into the region $x \rightarrow-\infty$. We are interested in the probability $\mathcal{P}(E, N)$ of reflection.

Let us discuss reflections at the classical level. [Note that the classical counterpart of $N$ is the energy of transverse oscillations.] Consider first the waveguide with one sharp turn [Fig. 1(a)]. One observes that the outcome of the classical evolution, i.e., whether or not the particle reflects from the turn, depends not only on the total energy $E$, but on other dynamical quantities as well. In particular, the direction of the momentum of the particle in the vicinity of the turn (point $C$ on the graph) is important. This means that the entire dynamics in the waveguide should be taken into account in order to determine the possibility of classical reflection. This is in sharp contrast with the situation in the onedimensional case, where reflection from the potential barrier (or transition through it) is ensured by the value of the conserved energy of the particle.

Now, consider the waveguide with two turns. The model is characterized by the angles of the turns and the distance $L$ between them [see Fig. 1(b)]. Suppose the particle starts moving classically from $x \rightarrow-\infty$ with $N=0$ along the valley $w=0$. Then, the transverse oscillations become excited only after the particle crosses the first turn, point $C^{\prime}$ on the plot, so that at the time of arrival to the second turn (point $C$ ) approximately $\omega \tau / 2 \pi$ oscillations are made, where $\tau$

\footnotetext{
${ }^{2}$ The explicit expressions for the waveguide functions $w(x, y)$ will be presented in the subsequent sections.
}

$\sim L \sqrt{m / 2 E}$ is the time of motion between the two turns. The state of the particle (coordinates and momenta) at which it comes across the second turn depends periodically on the phase of transverse oscillations $\omega \tau$. Hence, one expects that the regime of motion of the classical particle can change from transmission to reflection and back as the energy grows ( $\tau$ decreases); the energies where it happens can be roughly estimated as

$$
E_{n} \sim \frac{m \omega^{2} L^{2}}{2(2 \pi n)^{2}} .
$$

We will see that this is indeed the case for the waveguides with certain angles of the turns.

At some values of $E, N$ the reflection process cannot proceed classically. Then, at the quantum mechanical level its probability is exponentially suppressed, $F(E, N)>0$. It is natural to call such a process "overbarrier reflection" ${ }^{3}$. The central quantity to be studied below is the suppression exponent $F(E, N)$ of this process. The above discussion suggests that $F(E, N)$, being determined by the entire dynamics in the waveguide, may be a highly nontrivial function. For the particular case of the waveguide with alternating regimes of classical reflections and transmissions $F$ should oscillate: $F$ $=0$ at the energies where the classical reflections are allowed, and $F>0$ at the energies where the reflections are classically forbidden. One can expect that the similar oscillatory behavior of the suppression exponent persists for other two-turn models as well. Now, instead of reaching zero, $F$ may possess a number of local positive minima implying that the reflection at the optimal energies is still a tunneling process.

Let us emphasize the difference of the optimal tunneling from quantum interference and resonance phenomena in our two-turn model. The interference of the de Broglie waves reflected from the two turns can, in principle, lead to oscillations in the reflection probability $\mathcal{P}(E)$. One can estimate the positions of the interference peaks by equating the de Broglie wavelength of the particle to an integer fraction of the distance between the turns, $2 \pi \hbar / \sqrt{2 m E} \sim L / n$. This yields the energies of the interference peaks,

$$
E_{n}^{i n t} \sim \frac{(2 \pi n)^{2} \hbar^{2}}{2 m L^{2}} .
$$

This formula is completely different from Eq. (2) for the peaks due to optimal tunneling. In particular, the distance between the adjacent inteference peaks,

$$
\Delta E^{i n t} \sim \frac{2 \pi \hbar}{L} \sqrt{\frac{2 E}{m}},
$$

scales proportional to $\hbar$. Thus, these peaks should be averaged over in the semiclassical limit. Besides, the amplitude of the interference peaks is at most of order one and does not affect the suppression exponent. Indeed, the exponential increase of the scattering amplitude can arise due to quantum

\footnotetext{
${ }^{3}$ By this term we want to emphasize that the process is classically forbidden. Recall, however, that there is no actual potential barrier across the waveguide in our setup.
} 
interference only in the presence of a resonant state with exponentially long lifetime. This state should be supported somewhere in between the turns and should be classically stable. In Sec. IV B we show that such states are absent in our system. One concludes that the peaklike structure of the probability $\mathcal{P}(E)$ of optimal tunneling is caused by completely different physical reasons as compared to the case of resonance scattering in quantum theory.

It is worth noting that the phenomenon of optimal tunneling has an important implementation in field theory. Recently it was argued [17] (see also Ref. [16]) that the probability of tunneling induced by particle collisions $[18,19]$ reaches its maximum at a certain optimal energy and stays constant ${ }^{4}$ at higher energies. This result, if generic, provides the answer to the long-standing question [20] about the high-energy behavior of the probability of collision-induced nonperturbative transitions in field theory. The quantum-mechanical model presented here supports the generic nature of the phenomenon of optimal tunneling; the simplicity of our model enables one to obtain an intuitive insight into the nature of this phenomenon.

The paper is organized as follows. In Section II we review the semiclassical method of complex trajectories, which is exploited in the rest of the paper. Reflections in the waveguides with one and two turns are considered in Secs. III and IV, respectively. We discuss our results in Sec. V. In the Appendix we analyze the validity of some assumptions made in the main body of the paper.

\section{SEMICLASSICAL METHOD}

We start by describing the semiclassical method ${ }^{5}$ of complex trajectories which will be used in the study of overbarrier reflections. We concentrate on the derivation of the formula for the suppression exponent $F(E, N)$ (see Refs. [2,8,9] for the details of the method and Ref. [19] for the field theory formulation). In what follows we use the system of units

$$
\hbar=m=\omega=1,
$$

where the Hamiltonian takes the form

$$
H=\frac{1}{2}\left[p_{x}^{2}+p_{y}^{2}+w^{2}(x, y)\right]
$$

One starts with the amplitude of reflection into the state with definite coordinates $x_{f}<0, y_{f}$,

\footnotetext{
${ }^{4}$ As opposed to the quantum mechanical case, the tunneling probability does not decrease at energies higher than the optimal one. This is due to the possibility, specific to the field theoretical setup, to emit the excess of energy into a few hard particles, so that tunneling effectively occurs at the optimal energy.

${ }^{5}$ Note that the method has been confirmed by the explicit comparison with the exact quantum mechanical results in Refs. [8,9,14]; specifically, the recent check [14] deals with the case when the dependence of the suppression exponent on energy is not monotonic.
}

$$
\mathcal{A}=\left\langle x_{f}, y_{f}\left|e^{-i \hat{H}\left(t_{f}-t_{i}\right)}\right| E, N\right\rangle .
$$

Here $|E, N\rangle$ is the initial state of the particle moving in the asymptotic region $x_{i} \rightarrow-\infty$ with fixed translatory momentum $p_{0}=\sqrt{2(E-N)}$ and the oscillator excitation number $N$. Semiclassically,

$$
\left\langle x_{i}, y_{i} \mid E, N\right\rangle=e^{i p_{0} x_{i}} \cos \left(\int_{\sqrt{2 N}}^{y_{i}} p_{y}\left(y^{\prime}\right) d y^{\prime}+\pi / 4\right),
$$

where $x_{i}, y_{i}$ denote initial coordinates,

$$
p_{y}\left(y^{\prime}\right)=\sqrt{2 N-y^{\prime 2}},
$$

and we omitted the preexponential factor which is irrelevant for our purposes. Using Eq. (5), one rewrites the amplitude (4) as a path integral,

$$
\begin{aligned}
\mathcal{A}= & \left.\int d x_{i} d y_{i} \int[d x][d y]\right|_{x_{i}, y_{i}} ^{x_{f}, y_{f}} e^{i S+i p_{0} x_{i}} \\
& \times \cos \left(\int_{\sqrt{2 N}}^{y_{i}} p_{y}\left(y^{\prime}\right) d y^{\prime}+\pi / 4\right),
\end{aligned}
$$

where $S$ is the classical action of the model (3).

In the semiclassical case the integral (7) is dominated by the (generically complex) saddle point. Note that as we continue the integrand in Eq. (7) into the plane of complex coordinates, one of the exponents constituting the initial oscillator wave function grows, while the other becomes negligibly small. Within the validity of our approximation, we omit the decaying exponent by writing

$$
\cos \left(\int_{\sqrt{2 N}}^{y_{i}} p_{y}\left(y^{\prime}\right) d y^{\prime}+\pi / 4\right) \rightarrow \exp \left(i \int_{\sqrt{2 N}}^{y_{i}} p_{y}\left(y^{\prime}\right) d y^{\prime}\right),
$$

with the standard choice ${ }^{6}$ of the branch of the square root in Eq. (6).

One proceeds by finding the saddle point for the integral (7) with the substitution (8). Extremization with respect to $x(t), y(t)$ leads to the classical equations of motion,

$$
\ddot{x}=-w w_{x}, \quad \ddot{y}=-w w_{y} .
$$

Differentiating with respect to $x_{i} \equiv x\left(t_{i}\right), y_{i} \equiv y\left(t_{i}\right)$, one obtains

$$
\dot{x}_{i}=p_{0}=\sqrt{2(E-N)}, \quad \dot{y}_{i}=p_{y}\left(y_{i}\right)=\sqrt{2 N-y_{i}^{2}} .
$$

The latter equations are equivalent to fixing the total energy $E$ and initial oscillator energy $N$ of the complex trajectory,

$$
E=\frac{1}{2} \dot{x}_{i}^{2}+N
$$

\footnotetext{
${ }^{6}$ The correct branch is fixed by drawing a cut between the oscillator turning points $y= \pm \sqrt{2 N}$ and choosing $\operatorname{Im} p_{y}>0$ at $y \in \mathbb{R}, y$ $>\sqrt{2 N}$; see, e.g., Ref. [21].
} 


$$
N=\frac{1}{2}\left(\dot{y}_{i}^{2}+y_{i}^{2}\right)
$$

Substituting the saddle-point configuration ${ }^{7}$ into Eq. (7), one obtains the amplitude of the process with exponential accuracy,

$$
\mathcal{A} \propto e^{i S+i B\left(x_{i}, y_{i}\right)},
$$

where the term

$$
B\left(x_{i}, y_{i}\right)=p_{0} x_{i}+\int_{\sqrt{2 N}}^{y_{i}} p_{y}\left(y^{\prime}\right) d y^{\prime}
$$

is the initial-state contribution. For the inclusive reflection probability one writes

$$
\mathcal{P}=\int d x_{f} d y_{f}|\mathcal{A}|^{2} \propto \int d x_{f} d y_{f} e^{i S-i S^{*}+i B-i B^{*}} .
$$

The integral over the final states can also be evaluated by the saddle-point technique; extremization with respect to $x_{f}$ $\equiv x\left(t_{f}\right), \quad y_{f} \equiv y\left(t_{f}\right) \quad$ fixes the boundary conditions in the asymptotic future,

$$
\operatorname{Im} \dot{x}_{f}=\operatorname{Im} x_{f}=0, \quad \operatorname{Im} \dot{y}_{f}=\operatorname{Im} y_{f}=0 .
$$

In this way one obtains the expression (1) for the reflection probability, where the suppression exponent $F$ is given by the value of the functional

$$
F(E, N)=2 \operatorname{Im} S+2 \operatorname{Im} B\left(x_{i}, y_{i}\right)
$$

evaluated on the saddle-point configuration-a complex trajectory satisfying the boundary value problem (9), (10), (12).

The contribution $B\left(x_{i}, y_{i}\right)$ of the initial state is simplified after one uses the asymptotic form of the solution at $t \rightarrow-\infty\left(x_{i} \rightarrow-\infty\right)$

$$
x=p_{0} t+x_{0}, \quad y=a e^{-i t}+\bar{a} e^{i t} .
$$

Equations (10) guarantee that the quantities $p_{0}=\sqrt{2(E-N)}$ and $2 a \bar{a}=N$ are real, since $E, N \in \mathbb{R}$. Therefore, one may introduce two real parameters $T, \theta$ as follows:

$$
2 \operatorname{Im} x_{0}=-p_{0} T, \quad \bar{a}=a^{*} e^{T+\theta} .
$$

One finds for the initial term (11),

$$
\begin{aligned}
2 \operatorname{Im} B\left(x_{i}, y_{i}\right) & =\operatorname{Im}\left\{2 p_{0} x_{i}-2 N \arccos \left(y_{i} / \sqrt{2 N}\right)+y_{i} \sqrt{2 N-y_{i}^{2}}\right\} \\
& =-p_{0}^{2} T-N(T+\theta)+\operatorname{Im}\left(y_{i} \dot{y}_{i}\right),
\end{aligned}
$$

and thus

$$
F=2 \operatorname{Im} \tilde{S}-E T-N \theta
$$

where $\tilde{S}$ is the classical action of the system (3) integrated by parts,

$$
\tilde{S}=-\frac{1}{2} \int_{t_{i}}^{t_{f}} d t\left[x \ddot{x}+y \ddot{y}+w^{2}(x, y)\right] .
$$

\footnotetext{
${ }^{7}$ For simplicity we assume that the saddle-point configuration is unique. Otherwise, one should take the saddle point corresponding to the weakest exponential suppression.
}

Let us comment on the physical meaning of the parameters $T, \theta$. Consider two trajectories which are solutions to the boundary value problem (9), (10), (12) at neighboring values of $E, N$. The differential of the quantity $2 \operatorname{Im} \widetilde{S}$ as one deforms one trajectory into the other is

$$
\begin{aligned}
d(2 \operatorname{Im} \widetilde{S}) & =d \operatorname{Im}\left(2 S+x_{i} \dot{x}_{i}+y_{i} \dot{y}_{i}\right) \\
& =\operatorname{Im}\left(x_{i} d \dot{x}_{i}-\dot{x}_{i} d x_{i}+y_{i} d \dot{y}_{i}-\dot{y}_{i} d y_{i}\right) \\
& =E d T+N d \theta,
\end{aligned}
$$

where in the last equality we used the asymptotic form (13), (14) of the solution. Then, from Eq. (15) one finds

$$
d F(E, N)=-T d E-\theta d N .
$$

Thus, the parameters $T$ and $\theta$ are (up to sign) the derivatives of the suppression exponent with respect to energy $E$ and initial oscillator excitation number $N$, respectively.

Our final remark is that the boundary value problem (9), (10), (12) is invariant with respect to the trivial time translation symmetry,

$$
t \rightarrow t+\delta t, \quad \delta t \in \mathbb{R}
$$

which can be fixed in any convenient way.

\section{MODEL WITH ONE TURN}

To warm up, we consider the simplest model, where the waveguide has one sharp turn,

$$
\begin{aligned}
w= & y \theta(-x+y \tan \beta)+\cos \beta(x \sin \beta+y \cos \beta) \\
& \times \theta(x-y \tan \beta) .
\end{aligned}
$$

Here $\theta(x)$ is the step function. It is convenient to use the rotated coordinate system,

$$
\left(\begin{array}{l}
\xi \\
\eta
\end{array}\right)=\left(\begin{array}{cc}
\cos \beta & -\sin \beta \\
\sin \beta & \cos \beta
\end{array}\right)\left(\begin{array}{l}
x \\
y
\end{array}\right) .
$$

The waveguide function takes the form

$$
w=\eta \cos \beta-\xi \sin \beta \theta(-\xi) .
$$

The equipotential contour $w^{2}(\xi, \eta)=$ const is shown in Fig. 2 . One observes that the motion of the particle in two regions, $\xi<0$ and $\xi>0$, decomposes into the translatory motion and oscillations in the coordinates $x, y$ and $\xi, \eta$, respectively [see Eqs. (19) and (20)]; the frequency of $\eta$ oscillations in the latter case is $\cos \beta$.

Due to the presence of the step function, the first derivatives of the potential (20) are discontinuous ${ }^{8}$ at $\xi=0$. Strictly speaking, the semiclassical method is not applicable in this situation [21]. Thus, the formula (20) should be regarded as an approximation to some waveguide function with smooth turn. Generically the width of the smoothened turn is characterized by a parameter $b$; the sharp-turn approximation (20) corresponds to $b \rightarrow 0$. An example of smoothening is provided by the following substitution in Eq. (20),

\footnotetext{
${ }^{8}$ Note that the potential itself is continuous.
} 


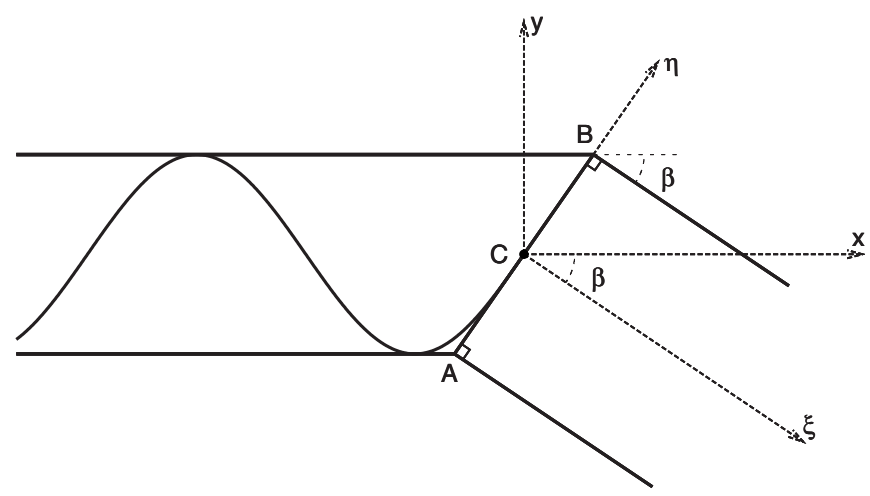

FIG. 2. The equipotential contour $w^{2}(x, y)=2 N$ for the waveguide (20) and the trajectory of the critical solution with energy $N / \cos ^{2} \beta$.

$$
\theta(\xi) \rightarrow \theta_{b}(\xi)=\frac{1}{1+e^{-\xi / b}}
$$

The semiclassical description can be used as long as the de Broglie wavelength of the particle is small compared to the linear size of the potential ${ }^{9}, 1 / \sqrt{E} \ll b$. We conclude that the sharp-turn and semiclassical approximations are valid simultaneously for smooth waveguides with

$$
1 \gg b \gg 1 / \sqrt{E} \text {. }
$$

An important property of the model (20) is invariance of the classical equations of motion (9) under the rescaling of the coordinates,

$$
x \rightarrow \Lambda x, \quad y \rightarrow \Lambda y .
$$

Using the transformation (23), one may express a solution $x(t), y(t)$ with energy $E$ in terms of the normalized one,

$$
x=\tilde{x} \sqrt{E}, \quad y=\tilde{y} \sqrt{E},
$$

where the solution $\tilde{x}(t), \tilde{y}(t)$ has unit energy; its initial oscillator excitation number is

$$
\nu=N / E \text {. }
$$

The suppression exponent (15) takes the form,

$$
F(E, N)=E f_{\beta}(\nu),
$$

where $f_{\beta}(\nu)$ is the exponent for the normalized solution. Substituting the expression (24) into Eq. (17), one obtains

$$
f_{\beta}(\nu)=-T-\theta \nu .
$$

We will exploit Eq. (25) in the end of this section. Now, we proceed to finding the normalized trajectories.

At certain initial data $\nu>\nu_{\text {cr }}$ the particle can reflect from the turn classically, so that

\footnotetext{
${ }^{9}$ Another semiclassical condition is that the energy is sufficient to excite a lot of oscillator levels, $E \gg 1$. It is satisfied provided Eq. (22) holds.
}

$$
f_{\beta}\left(\nu>\nu_{\mathrm{cr}}\right)=0 .
$$

Let us find the value of $\nu_{\mathrm{cr}}$. In the region $\xi<0$ the classical solution takes the form

$$
\begin{gathered}
x(t)=p_{0} t+x_{0}, \\
y(t)=A_{0} \sin (t+\varphi) .
\end{gathered}
$$

Having crossed the line $\xi=0$ (line $A B$ in Fig. 2), the classical particle can never return back into the region $\xi<0$. Indeed, in this case it moves at $\xi>0$ with constant momentum $p_{\xi}$ $>0$. Thus, the particle can reflect classically only if its trajectory touches the line $\xi=0$. The potential of our model has ill-defined derivatives at $\xi=0$, and the fate of the particle moving along the line $A B$ depends on the particular choice of the smoothening of the potential. In the Appendix we consider the motion of the classical particle in the case when nonzero smoothening of width $b$ is switched on. For a class of smoothenings we show that in the small vicinity $(\delta \xi$ $\sim b$ ) of any trajectory touching the line $\xi=0$ there exists some "smoothened" trajectory, which reflects classically from the turn. Consequently, below we associate the trajectories touching the line $\xi=0$ with the classical reflected solutions.

One notices that the inclination of the trajectory (26) is bounded from above,

$$
\left|\frac{d y}{d x}\right| \leq \frac{A_{0}}{p_{0}} ;
$$

therefore, the classical trajectory of the particle can touch the line $\xi=0$, that is, $y / x=\cot \beta$ only at

$$
A_{0} / p_{0} \geq \cot \beta \text {. }
$$

From Eqs. (27), (26), and (10) one extracts the condition for the particle to reflect classically from the turn,

$$
\nu \geq \nu_{\mathrm{cr}}=\cos ^{2} \beta \text {. }
$$

The critical classical solution at $\nu=\nu_{\mathrm{cr}}$ touches the line $\xi=0$ at $\eta=0$ (point $C$ in Fig. 2), where its trajectory

$$
\begin{gathered}
x_{\mathrm{cr}}(t)=\sqrt{2} t \sin \beta, \\
y_{\mathrm{cr}}(t)=\sqrt{2} \sin t \cos \beta .
\end{gathered}
$$

has the largest inclination.

We now turn to the classically forbidden reflections at $\nu$ $\left\langle\nu_{\text {cr }}\right.$, which are described by the boundary value problem (9), (10), (12). One makes the following important observation. The waveguide function (20) has the form of two analytic functions glued together at $\xi=0$. Hence, the equations of motion (9) can be continued analytically to the complex values of coordinates in two different ways, starting from the regions $\xi<0$ and $\xi>0$, respectively. In this way one obtains two complex solutions, $\xi_{-}(t), \eta_{-}(t)$ and $\xi_{+}(t), \eta_{+}(t)$. These solutions and their first derivatives should be matched at some moment of time $t_{1}, \xi\left(t_{1}\right)=0$. [Note that the matching time $t_{1}$ does not need to be real.] Below we conventionally refer to these solutions as the ones belonging to the regions $\xi<0$ and $\xi>0$. 
By the same reasoning as above we find that once the particle arrives into the region $\xi>0$, it never reflects back to $\xi<0$, unless $p_{\xi}=0$. So, in the region $\xi>0$ one writes

$$
\begin{gathered}
\xi_{+}(t)=0, \\
\eta_{+}(t)=\frac{\sqrt{2}}{\cos \beta} \sin \left(t \cos \beta+\varphi_{\eta}\right),
\end{gathered}
$$

where the normalization condition $E=1$ has been used explicitly. Due to the conditions in the asymptotic future, Eqs. (12), the parameter $\varphi_{\eta}$ is real. We use the translational invariance (18) to set $\varphi_{\eta}=0$. Note that we again associate the trajectory going along the line $\xi=0$ with the reflected one.

The physical picture of overbarrier reflection that comes to mind matches with the mechanism of multidimensional tunneling proposed recently in Refs. $[9,11]$. The process proceeds in two steps. The first step, which is exponentially suppressed, is the formation of the periodic classical orbit (30) oscillating along the line $\xi=0$. This orbit is unstable. At the second step of the process the unstable orbit decays classically forming a trajectory going back to $x \rightarrow-\infty$ at $t \rightarrow$ $+\infty$. Clearly, the second step does not affect the suppression exponent of the whole process, and we do not consider it explicitly. In what follows we concentrate on the determination of the tunneling trajectory describing the first step of the process.

One should find the solution at $\xi<0$ and impose the boundary conditions (10). Note, however, that the energy of our solution is fixed already. As for the initial oscillator excitation number $\nu$, it does not change during the evolution in the region $\xi<0$. Thus, one may fix it at the matching time $t=t_{1}$. One writes

$$
\nu=\left.\frac{1}{2}\left(\dot{y}^{2}+y^{2}\right)\right|_{t=t_{1}}=\cos ^{2} \beta+\sin ^{2} \beta \sin ^{2}\left(t_{1} \cos \beta\right) .
$$

This complex equation allows one to express $t_{1}$ as

$$
\sin \left(t_{1} \cos \beta\right)=-i \frac{\sqrt{\nu_{\mathrm{cr}}-\nu}}{\sin \beta},
$$

where the choice of the sign is dictated by the condition in footnote ${ }^{6}$. It is convenient to introduce notation $t_{1}=i T_{1}, T_{1}$ $\in \mathbb{R}$.

In order to find the suppression exponent $f_{\beta}(\nu)$, one needs to evaluate the parameters $T(\nu), \theta(\nu)$. At $\xi<0$ the solution has the form

$$
\begin{aligned}
& x_{-}(t)=p_{0}(t-i T / 2)+x_{0}^{\prime}, \\
& y_{-}(t)=a e^{-i t}+a^{*} e^{T+\theta+i t},
\end{aligned}
$$

where the definitions (13) and (14) have been taken into account explicitly, so that $p_{0}, x_{0}^{\prime} \in \mathbb{R}$. One evaluates $p_{0}, x_{0}^{\prime}, a$, $T, \theta$ by matching the coordinates $x_{ \pm}, y_{ \pm}$and their first derivatives $\dot{x}_{ \pm}, \dot{y}_{ \pm}$at $t=i T_{1}$; this yields

$$
\begin{gathered}
x_{0}^{\prime}=0, \quad p_{0}=\sqrt{2(1-\nu)}, \\
a=i \sqrt{\frac{\nu}{2}} e^{-(T+\theta) / 2},
\end{gathered}
$$

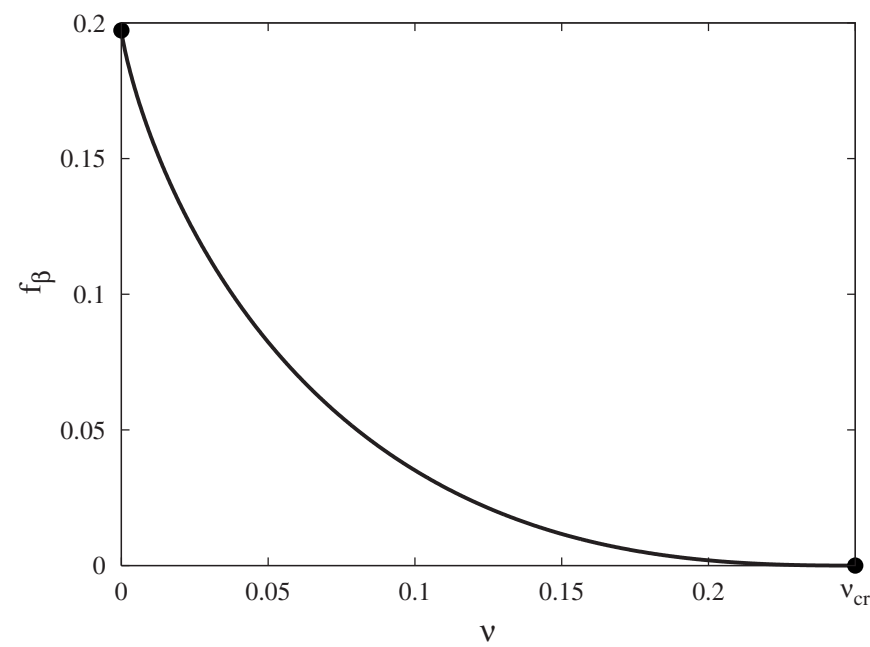

FIG. 3. The suppression exponent $f_{\beta}(\nu)$ for the waveguide (20); $\beta=\pi / 3$.

$$
\begin{gathered}
T_{1}-\frac{T}{2}=-\sqrt{\frac{1-\nu / \cos ^{2} \beta}{1-\nu},} \\
\sinh \left(T_{1}-\frac{T+\theta}{2}\right)=-\frac{\sqrt{\cos ^{2} \beta-\nu}}{\sin \beta \sqrt{\nu}} .
\end{gathered}
$$

The last two equations, together with Eq. (25), define the function $f_{\beta}(\nu)$,

$$
\begin{aligned}
f_{\beta}(\nu)= & \frac{2}{\cos \beta}\left\{\operatorname{arcsinh} \frac{\sqrt{\nu_{\mathrm{cr}}-\nu}}{\sin \beta}-\nu \cos \beta \operatorname{arcsinh} \frac{\sqrt{\nu_{\mathrm{cr}}-\nu}}{\sin \beta \sqrt{\nu}}\right. \\
& \left.-\sqrt{\left(\nu_{\mathrm{cr}}-\nu\right)(1-\nu)}\right\} ;
\end{aligned}
$$

this function is plotted in Fig. 3. One observes that at $\nu$ $\rightarrow \nu_{\text {cr }}$ the quantities $T_{1}, T, \theta, f_{\beta}$ tend to zero, and the complex trajectory tends to the classically allowed critical solution, cf. Eqs. (29),

$$
p_{0} \rightarrow \sqrt{2} \sin \beta, \quad a \rightarrow \frac{i}{\sqrt{2}} \cos \beta .
$$

At $\nu=0$ one has

$$
f_{\beta}(0)=-2+\frac{2}{\cos \beta} \operatorname{arctanh}(\cos \beta) .
$$

To summarize, we obtained the suppression exponent for the reflection of a particle in the simplest waveguide with one sharp turn.

\section{MODEL WITH TWO TURNS}

\section{A. Introducing the system}

In the model of the previous section the suppression exponent was proportional to energy because of the coordinate rescaling symmetry (23). Now, we are going to demonstrate that a small violation of this symmetry results in a highly nontrivial graph for $F(E)$. 


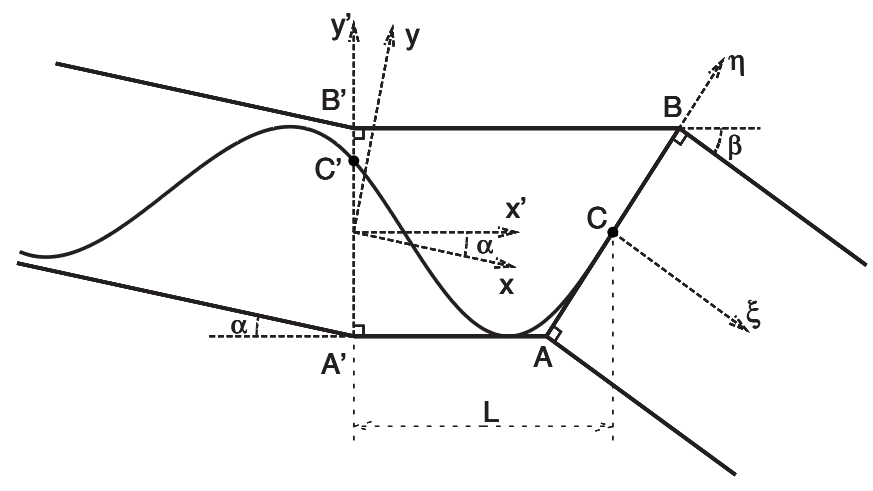

FIG. 4. The equipotential contour $w^{2}(x, y)=2 N^{\prime}$ for the waveguide (35) and the trajectory of the critical solution with energy $N^{\prime} / \cos ^{2} \beta>E_{B}$. The matching points $C, C^{\prime}$ are shown by the thick black dots.

One introduces a second turn into the waveguide, see Fig. 4. We want to consider this turn as a small perturbation, so, we assume its angle $\alpha$ to be smaller than $\beta$. It is convenient to introduce two additional coordinate systems, $x^{\prime}, y^{\prime}$ and $\xi$, $\eta$, bound to the central and rightmost parts of the waveguide, respectively. They are related to the original coordinate system $x, y$ as follows,

$$
\begin{aligned}
& \left(\begin{array}{l}
x^{\prime} \\
y^{\prime}
\end{array}\right)=\left(\begin{array}{cc}
\cos \alpha & \sin \alpha \\
-\sin \alpha & \cos \alpha
\end{array}\right)\left(\begin{array}{l}
x \\
y
\end{array}\right), \\
& \left(\begin{array}{l}
\xi \\
\eta
\end{array}\right)=\left(\begin{array}{cc}
\cos \beta & -\sin \beta \\
\sin \beta & \cos \beta
\end{array}\right)\left(\begin{array}{c}
x^{\prime}-L \\
y^{\prime}
\end{array}\right) .
\end{aligned}
$$

Note that the origin of the coordinate system $\xi, \eta$ is shifted by the distance $L$. The waveguide function is

$$
\begin{aligned}
w= & \theta\left(-x^{\prime}\right) \theta(-\xi) y+\theta(-\xi) \theta\left(x^{\prime}\right) y^{\prime} \cos \alpha \\
& +\theta(\xi) \eta \cos \alpha \cos \beta
\end{aligned}
$$

it consists of three pieces glued together continuously at $x^{\prime}$ $=0$ and $\xi=0$ (lines $A^{\prime} B^{\prime}$ and $A B$ in Fig. 4, respectively). At $t \rightarrow-\infty$ the particle comes flying from the asymptotic region $x^{\prime}<0$, where $w=y$. In the intermediate region $x^{\prime}>0, \xi<0$ the particle moves in the $x^{\prime}$ direction oscillating along the $y^{\prime}$ coordinate with the frequency $\cos \alpha$. Finally, in the region $\xi>0$ its motion is free in the coordinates $\xi, \eta$; the frequency of $\eta$ oscillations is $\cos \alpha \cos \beta$.

The model (35) no longer possesses the symmetry (23): rescaling of coordinates changes the length $L$ of the central part of the waveguide. In what follows it is convenient to work in terms of the rescaled dynamical variables,

$$
\tilde{x}=x / L, \quad \tilde{y}=y / L .
$$

In these terms the parameter $L$ disappears from the classical equations of motion, entering the theory through the overall coefficient $L^{2}$ in front of the action. The initial-state quantum numbers are also proportional to $L^{2}$,

$$
E=L^{2} \widetilde{E}, \quad N=L^{2} \tilde{N}
$$

Thus, the conditions (22) for the validity of the semiclassical approximation are satisfied in the limit

$$
L \rightarrow \infty, \quad \tilde{E}, \tilde{N}=\text { fixed }
$$

The suppression exponent takes the form

$$
F(E, N)=L^{2} \widetilde{F}(\widetilde{E}, \tilde{N}) .
$$

To simplify notations, we omit tildes over the rescaled quantities in the rest of this section. Rescaling back to the physical units can be easily performed in the final formulas by implementing Eqs. (36) and (37).

\section{B. Classical evolution}

Let us begin this section by demonstrating that there are no stable classical solutions localized in the region between the turns. This is important for the determination of the tunneling probability, since such stable solutions could lead to exponential resonances in the tunneling amplitude. The argument proceeds as follows. Any trajectory which is localized in the intermediate region should reflect from the line $A B$ infinitely many times. Each reflection involves touching the unstable orbit living at the line $A B$. This implies that the trajectory itself is unstable.

We proceed by determining the region of initial data $E, N$, which correspond to the classical reflections. (For brevity we will refer to this region as the "classically allowed region," as opposed to the "classically forbidden region" where reflections occur only at the quantum mechanical level. We stress that these are the regions in the plane of quantum numbers $E, N$.) Let us search for the critical classical solutions which correspond to the smallest initial oscillator number $N=N_{\mathrm{cr}}(E)$ at given energy $E$. As in the previous section, one finds that the particle must stick at the line ${ }^{10} A B$ for some time in order to reflect back. Let us first make an assumption inspired by the study of the one-turn model that the critical solutions touch the line $A B$ at their maximum inclination point (point $C$ in Fig. 4). We will see shortly that this is true only at energies above a certain value $E_{B}$, see Eq. (50). Still, the analysis based on the above assumption enables one to catch the qualitative features of the critical line $N=N_{\text {cr }}(E)$. Besides, the analysis is considerably simplified in this case; we postpone the accurate study until the end of this section. Keeping in mind the above remarks, one writes for the solution in the intermediate region,

$$
\begin{gathered}
x_{\mathrm{cr}}^{\prime}(t)=t \sqrt{2 E} \sin \beta+1, \\
y_{\mathrm{cr}}^{\prime}(t)=\sqrt{2 E} \frac{\cos \beta}{\cos \alpha} \sin (t \cos \alpha) .
\end{gathered}
$$

Before entering the intermediate region, the particle crosses the line $A^{\prime} B^{\prime}$ (point $C^{\prime}$ in Fig. 4). The initial oscillator num-

\footnotetext{
${ }^{10} \mathrm{We}$ do not consider reflections from the line $A^{\prime} B^{\prime}$. They disappear at larger values of $N$ than reflections from the line $A B$ if $\alpha$ is small enough.
} 


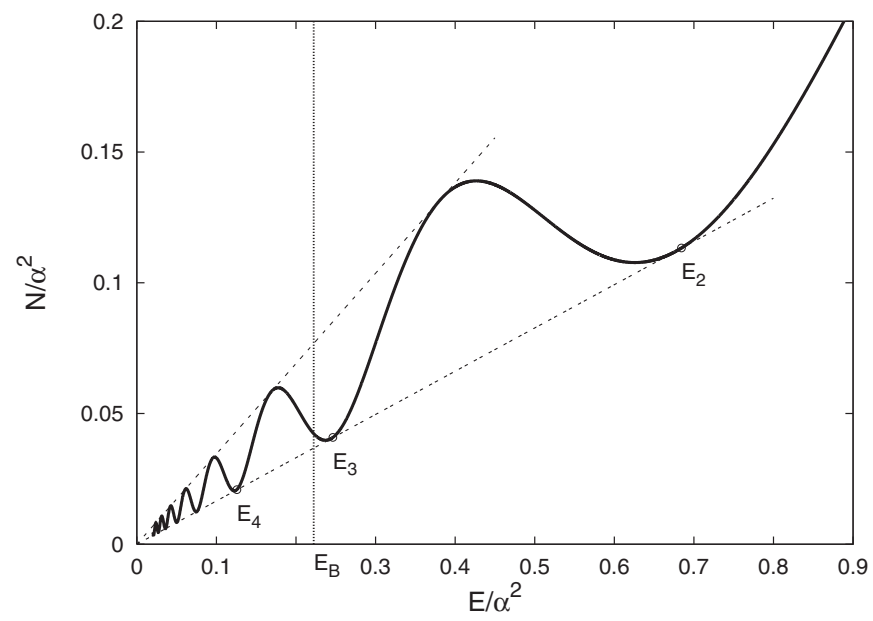

FIG. 5. The boundary $N=N_{\mathrm{cr}}(E)$ of the classically allowed region at $E>E_{B}$ for the waveguide model (35); $\beta=\pi / 3, \alpha=\pi / 30$. The region of the classically allowed initial data lies above this boundary. The empty circles correspond to the energies $E=E_{n}$, where the curve $N=N_{\mathrm{cr}}(E)$ touches its lower envelope $N$ $=E \cos ^{2}(\beta+\alpha)$.

ber $N$ is most conveniently calculated at the moment

$$
t=t_{0} \equiv-\frac{1}{\sqrt{2 E} \sin \beta}
$$

of crossing. Using the relations (34) one obtains

$$
\dot{x}_{\mathrm{cr}}\left(t_{0}\right)=\sqrt{2 E}\left[\sin \beta \cos \alpha-\cos \beta \sin \alpha \cos \left(\frac{\cos \alpha}{\sqrt{2 E} \sin \beta}\right)\right],
$$

and thus

$$
\begin{aligned}
N_{\mathrm{cr}}(E)= & E-\frac{1}{2} \dot{x}_{\mathrm{cr}}^{2}\left(t_{0}\right)=E-E[\sin \beta \cos \alpha \\
& \left.-\cos \beta \sin \alpha \cos \left(\frac{\cos \alpha}{\sqrt{2 E} \sin \beta}\right)\right]^{2}, \quad E>E_{B}
\end{aligned}
$$

As an example, we show in Fig. 5 the region of the classically allowed initial data for $\beta=\pi / 3, \alpha=\pi / 30$. One observes that the function $N_{\mathrm{cr}}(E)$ oscillates between two linear envelopes, $E \cos ^{2}(\beta+\alpha)$ and $E \cos ^{2}(\beta-\alpha)$; the period of oscillations decreases as $E \rightarrow 0$. Moreover, the curve $N_{\text {cr }}(E)$ has a number of minima at the points $E=E_{n}^{\mathrm{cr}}$. This means that the energies $E=E_{n}^{\mathrm{cr}}$ are optimal for reflection: in the vicinity of any point $E=E_{n}^{\mathrm{cr}}, N=N_{\mathrm{cr}}\left(E_{n}^{c r}\right)$ reflections become exponentially suppressed independently of whether the energy is increased or decreased. This feature is particularly pronounced in the case $\alpha+\beta=\pi / 2$, when the lower envelope coincides with the line $N=0$. Then, the classical reflections (i.e., reflections with the probability of order 1 )at $N=0$ are possible only in the vicinities of the points

$$
E=\frac{1}{8 \pi^{2}(n-1 / 2)^{2}}
$$

This is the case we used in the Introduction to illustrate the effect.

The minima $E=E_{n}^{\mathrm{cr}}$ exist at other values of the parameters as well. For instance, let us find the positions of these minima in the case $\alpha \ll 1$. One differentiates Eq. (40) with respect to energy and obtains

$$
E_{n}^{\mathrm{cr}}=E_{n}\left\{1-\frac{1}{\pi(n-1 / 2)} \arcsin \left(\frac{\cot \beta}{2 \pi \alpha(n-1 / 2)}\right)+O\left(\alpha^{2}\right)\right\},
$$

where

$$
E_{n}=\frac{1}{8 \pi^{2}(n-1 / 2)^{2} \sin ^{2} \beta}
$$

are the points where the curve $N=N_{\mathrm{cr}}(E)$ touches its lower envelope. The argument of arcsine in Eq. (41) should be smaller than one, so, the minima $E_{n}^{\text {cr }}$ exist only at large enough $n$,

$$
n \geq n_{0} \equiv\left[\frac{\cot \beta}{2 \pi \alpha}+\frac{1}{2}\right]+1
$$

where [.] stands for the inteter part.

Let us make several comments. First, note that $n_{0}$ $\sim O(1 / \alpha)$, consequently, all the optimal points $E_{n}^{\mathrm{cr}}$ lie in the region of small energies $E \sim 1 / n_{0}^{2} \sim O\left(\alpha^{2}\right)$. Second, as we pointed out before, the formula (40) for the function $N_{\mathrm{cr}}(E)$ holds at $E>E_{B}$. Comparing the expressions (42), (43), and (50), one observes that $E_{n_{0}}>E_{B}$ if $\tan \beta>1$. So, there does exist a range of energies where the nonmonotonic behavior of the function $N_{\mathrm{cr}}(E)$ can be inferred from the formula (40). In fact, the conclusion about the existence of the local minima of $N_{\mathrm{cr}}(E)$, as well as the expressions (41)-(43) determining their positions, remain valid also at $E<E_{B}$. This follows from the rigorous analysis of the boundary of the classically allowed region to which we turn now. The reader who is more interested in the tunneling processes may skip this part and proceed directly to Sec. IV C.

Now, we do not appeal to the ansatz (38). Instead, we start with the general solution in the intermediate region,

$$
\begin{gathered}
x^{\prime}=p_{0}^{\prime}\left(t-t_{0}\right), \\
y^{\prime}=A_{0}^{\prime} \sin \left[\left(t-t_{0}\right) \cos \alpha+\varphi^{\prime}\right] .
\end{gathered}
$$

It is convenient to parametrize it by the total energy $E$ $=p_{0}^{\prime 2} / 2+\cos ^{2} \alpha A_{0}^{\prime 2} / 2$ and the "inclination" $\gamma$ defined by the relation

$$
p_{0}^{\prime} / A_{0}^{\prime}=\tan \gamma \cos \alpha .
$$

Expressions (44) take the following form:

$$
x^{\prime}=\sqrt{2 E}\left(t-t_{0}\right) \sin \gamma,
$$




$$
y^{\prime}=\sqrt{2 E} \frac{\cos \gamma}{\cos \alpha} \sin \left[\left(t-t_{0}\right) \cos \alpha+\varphi^{\prime}\right]
$$

The constants $t_{0}$ and $\varphi^{\prime}$ are fixed by demanding the trajectory (45) to reflect classically from the second turn, i.e., touch the line $\xi=0$ at $t=0$,

$$
\left(x^{\prime}-1\right) \cos \beta-\left.y^{\prime} \sin \beta\right|_{t=0}=0,\left.\quad \frac{d y^{\prime}}{d x^{\prime}}\right|_{t=0}=\cot \beta .
$$

These conditions imply

$$
\begin{gathered}
t_{0}=-\frac{1}{\sqrt{2 E} \sin \gamma}+\frac{1}{\cos \alpha} \sqrt{\frac{\tan ^{2} \beta}{\tan ^{2} \gamma}-1,} \\
\varphi^{\prime}=-\frac{\cos \alpha}{\sqrt{2 E} \sin \gamma}+\sqrt{\frac{\tan ^{2} \beta}{\tan ^{2} \gamma}-1}-\arccos \frac{\tan \gamma}{\tan \beta} .
\end{gathered}
$$

One sees that the classical reflections are possible only at $\gamma$ $\in[0 ; \beta]$; the boundary value $\gamma=\beta$ reproduces the solution (38).

In order to find $N_{\mathrm{cr}}(E)$, one should minimize the value of the incoming oscillator excitation number with respect to $\gamma$ at fixed $E$. At $t=t_{0}$, when the particle crosses the first turn,

$$
p_{0} \equiv \dot{x}\left(t_{0}\right)=\sqrt{2 E}\left(\cos \alpha \sin \gamma-\sin \alpha \cos \gamma \cos \varphi^{\prime}\right) \text {. }
$$

Since $N=E-p_{0}^{2} / 2$, one can maximize the value of the translatory momentum $p_{0}$ instead of minimizing $N(\gamma)$. Formula (39) represents the value $\gamma=\beta$ lying at the boundary of the accessible $\gamma$ domain; this value should be compared to $p_{0}(\gamma)$ taken at local maxima.

Let us consider the case $\alpha \ll 1$. At large enough energies, $E \sim 1$, Eq. (47) is dominated by the first term, which grows with $\gamma$, so that the maximum of $p_{0}(\gamma)$ is indeed achieved at $\gamma=\beta$. At small energies, however, the second term in Eq. (47) becomes essential because of the quickly oscillating $\cos \varphi^{\prime}$ multiplier: the frequency of $\cos \varphi^{\prime}$ oscillations grows as $E \rightarrow 0$, and at $E \sim \alpha^{2}$, in spite of the small magnitude proportional to $\sin \alpha$, the second term produces the sequence of local maxima of the function $p_{0}(\gamma)$.

One expects the parameters of the trajectory at small $\alpha$ not to be very different from the ones at $\alpha=0$ (the latter case was considered in Sec. III). So, we write

$$
\gamma=\beta-\delta \gamma
$$

where $0<\delta \gamma \ll 1$. Expanding the expressions (46) and (47) and taking into account that $E \sim \alpha^{2}$, one obtains

$$
\begin{gathered}
\varphi^{\prime}=-\frac{1}{\sqrt{2 E} \sin \beta}(1+\delta \gamma \cot \beta), \\
p_{0}=\sqrt{2 E}\left(\sin \beta-\delta \gamma \cos \beta-\alpha \cos \beta \cos \varphi^{\prime}\right) .
\end{gathered}
$$

Now, the local maxima of the initial translatory momentum can be obtained explicitly by differentiating Eqs. (48) with respect to $\delta \gamma$. One finds the sequence of them,

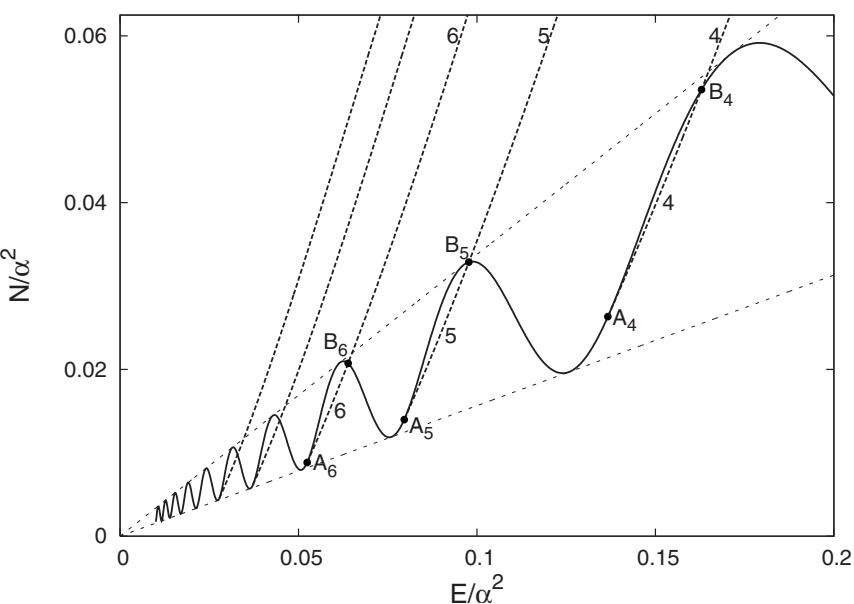

FIG. 6. The graphs $N_{n}(E)$ corresponding to the local minima of the function $N(\gamma)$ (dashed lines) plotted together with the "global" curve, Eq. (40) (solid line); $\beta=\pi / 3, \alpha=\pi / 30$. The critical curve $N=N_{\mathrm{cr}}(E)$ is obtained by taking the minimum among all the graphs.

$$
\begin{aligned}
\delta \gamma_{n}= & -\tan \beta+\sqrt{2 E} \frac{\sin ^{2} \beta}{\cos \beta} \\
& \times\left[2 \pi n-\pi-\arcsin \left(\frac{\sqrt{2 E} \sin ^{2} \beta}{\alpha \cos \beta}\right)\right] .
\end{aligned}
$$

Only the maxima with $\delta \gamma_{n}>0$ should be taken into account. The local maxima exist when

$$
E \leq E_{B} \equiv \frac{\alpha^{2} \cos ^{2} \beta}{2 \sin ^{4} \beta} .
$$

Substituting Eq. (49) into the expressions (48), one evaluates the values of $p_{0}$ at the local maxima,

$$
\begin{aligned}
p_{0, n}(E)= & 2 \sqrt{2 E} \sin \beta-2 E \sin ^{2} \beta \\
& \times\left[2 \pi n-\pi-\arcsin \left(\frac{\sqrt{2 E} \sin ^{2} \beta}{\alpha \cos \beta}\right)\right] \\
& +\alpha \sqrt{2 E} \cos \beta \sqrt{1-\frac{2 E \sin ^{4} \beta}{\alpha^{2} \cos ^{2} \beta}} .
\end{aligned}
$$

The graphs $N_{n}(E)=E-p_{0, n}^{2}(E) / 2$ are shown in Fig. 6 for the case $\beta=\pi / 3, \alpha=\pi / 30$. Each graph is plotted for the energy range $E>E_{A_{n}}$ restricted by condition $\delta \gamma_{n}>0$. They are presented together with the curve given by the formula (40). By definition, the critical solution corresponds to the lowest of these graphs. Clearly, for each "local" curve representing the $n$th local minimum of $N(\gamma)$ there is a range of energies $E_{A_{n}}$ $<E<E_{B_{n}}$ where it lies lower than the "global" curve (40). This means that the parameter $\gamma$ of the critical solution changes discontinuously across the points $E=E_{B_{n}}$. Correspondingly, the curve $N_{\mathrm{cr}}(E)$ has a break at these points. On the other hand, the function $N_{\mathrm{cr}}(E)$ is smooth at the points $A_{n}$ as the local graphs end up exactly at $\delta \gamma=0$, where the parameters of the $n$th local solution coincide with the ones of the global solution. 
To summarize, we have observed that the boundary of the classically allowed region is given by a collection of many branches of classical solutions, each branch being relevant in its own energy interval. We will see that a similar branch structure is present in the complex trajectories describing overbarrier reflections in the classically forbidden region of $E, N$.

\section{Classically forbidden reflections}

In this section we demonstrate that the suppression exponent $F(E, N)$ viewed as a function of energy at fixed $N$ exhibits oscillations deep inside the classically forbidden region of initial data. This result comes without surprise if one takes into account the nonmonotonic behavior of the boundary $N_{\mathrm{cr}}(E)$ of the classically allowed region. Indeed, the curve $N=N_{\mathrm{cr}}(E)$ coincides with the line $F(E, N)=0$. One has,

$$
\frac{d N_{\mathrm{cr}}}{d E}=-\left.\frac{\partial_{E} F}{\partial_{N} F}\right|_{N=N_{\mathrm{cr}}(E)},
$$

so that

$$
\frac{\partial F}{\partial E}\left(E_{n}^{\mathrm{cr}}, N_{n}^{\mathrm{cr}}\right)=0
$$

We conclude that the points $E=E_{n}^{\mathrm{cr}}$ are the local minima of the function $F(E)$ at fixed $N=N_{n}^{\mathrm{cr}}$. It is natural to expect that such local minima of $F(E)$ exist at other values of $N$ as well. To illustrate this fact explicitly, we study the complex trajectories, solutions to Eqs. (9), (10), and (12).

Following the tactics of the previous section, we find solutions in three separate regions: initial region $x^{\prime}<0$, final region $\xi>0$, and the intermediate region $x^{\prime}>0, \xi<0$. These solutions, together with their first derivatives, should be glued at $t=t_{0}$, when the complex trajectory crosses the line $x^{\prime}=0$, and at $t=t_{1}$, when $\xi=0$. Besides, we are looking for the tunneling solution which ends up oscillating along the line $A B$, see Fig. 4. As discussed in Sec. III this assumes existence of the second step of the process: classical decay of the unstable orbit living at $\xi=0$; the latter decay is described by a real trajectory ${ }^{11}$ going to $x \rightarrow-\infty$ at $t \rightarrow+\infty$.

The solution in the final region $\xi>0$ is [cf. Eqs. (30)]

$$
\begin{gathered}
\xi_{+}(t)=0, \\
\eta_{+}(t)=\frac{\sqrt{2 E}}{\cos \alpha \cos \beta} \sin (t \cos \alpha \cos \beta),
\end{gathered}
$$

where we used the time translation invariance (18) to fix the final oscillator phase $\varphi_{\eta}=0$. In the intermediate region $x^{\prime}$ $>0, \xi<0$ one writes

\footnotetext{
${ }^{11}$ One wonders why this trajectory does not reflect from the turn $A^{\prime} B^{\prime}$ on its way back. This concern is removed by the observation that the trajectory produced in the decay of the unstable orbit is not unique: in the Appendix we show that the decay can occur at any point of the segment $A C$ giving rise to a whole bunch of potential decay trajectories. Most of these trajectories pass through the turn $A^{\prime} B^{\prime}$ without reflection.
}

$$
\begin{gathered}
x^{\prime}(t)=p_{0}^{\prime} t+x_{0}^{\prime}, \\
y^{\prime}(t)=a^{\prime} e^{-i t \cos \alpha}+\bar{a}^{\prime} e^{i t \cos \alpha} .
\end{gathered}
$$

Note that the final solution (51) does not contain free parameters; thus, the matching of $x^{\prime}, \dot{x}^{\prime}, y^{\prime}, \dot{y}^{\prime}$ at $t=t_{1}$ enables one to express all the parameters in Eqs. (52) in terms of one complex variable $t_{1}$,

$$
\begin{gathered}
p_{0}^{\prime}=\sqrt{2 E} \sin \beta \cos \phi_{1}, \\
x_{0}^{\prime}=1+\sqrt{2 E} \frac{\tan \beta}{\cos \alpha}\left(\sin \phi_{1}-\phi_{1} \cos \phi_{1}\right), \\
a^{\prime}=\frac{\sqrt{E / 2}}{\cos \alpha} e^{i \phi_{1} / \cos \beta}\left(\sin \phi_{1}+i \cos \beta \cos \phi_{1}\right), \\
\bar{a}^{\prime}=\frac{\sqrt{E / 2}}{\cos \alpha} e^{-i \phi_{1} / \cos \beta}\left(\sin \phi_{1}-i \cos \beta \cos \phi_{1}\right),
\end{gathered}
$$

where we introduced $\phi_{1}=t_{1} \cos \alpha \cos \beta$.

As the energy of the solution has been fixed already, the only remaining initial condition involves initial oscillator excitation number at $x^{\prime}<0$, see Eqs. (10). It is convenient to impose this condition at the matching point $t=t_{0}$. One recalls the definition of the matching time $t_{0}$,

$$
p_{0}^{\prime} t_{0}+x_{0}^{\prime}=0
$$

which, after taking into account the expressions (53a) and (53b), leads to the following equation:

$$
\frac{\cos \alpha}{\sqrt{2 E} \sin \beta}+\frac{\sin \phi_{1}}{\cos \beta}-\cos \phi_{1} \Delta \phi=0,
$$

where $\Delta \phi=\cos \alpha\left(t_{1}-t_{0}\right)$. At $t=t_{0}$ one has

$$
\dot{x}\left(t_{0}\right)=p_{0}^{\prime} \cos \alpha-\dot{y}^{\prime}\left(t_{0}\right) \sin \alpha=\sqrt{2(E-N)},
$$

and thus

$$
\begin{aligned}
\frac{\sqrt{1-\nu}}{\sin \alpha}= & \cot \alpha \sin \beta \cos \phi_{1}-\sin \phi_{1} \sin \Delta \phi \\
& -\cos \beta \cos \phi_{1} \cos \Delta \phi .
\end{aligned}
$$

As before, $\nu=N / E$.

Two complex equations (54) and (55) determine the matching times $t_{0}, t_{1}$, and, consequently, the complex trajectory. Although these equations cannot be solved explicitly, they can be simplified in the case $\alpha \ll 1$, which we consider from now on. For concreteness, we study reflections at $N$ $=0$. It is important to keep in mind that in the region of interest $E \sim E_{n}^{\mathrm{cr}} \sim O\left(\alpha^{2}\right)$; thus, one should regard all the momenta $p$ and oscillator amplitudes $a, \bar{a}$, as the quantities of order $O(\alpha)$. At the same time, for the distances along the waveguide one has $x \sim O(1)$, so that the real parts of time intervals may be parametrically large, $\operatorname{Re} t \sim x / p \sim O(1 / \alpha)$.

Further on, it will be convenient to work in terms of real variables, so, we represent $\phi_{1}$ and $\Delta \phi$ as 


$$
\phi_{1}=\cos \alpha \cos \beta\left(\tau_{1}+i T_{1}\right), \quad \Delta \phi=\cos \alpha(\tau+i \Delta T) .
$$

Note that $\tau$ and $\Delta T$ are the real and imaginary parts of the time interval $t_{1}-t_{0}$ which the particle spends in the intermediate region. Now, Eq. (54) enables one to express

$$
\begin{gathered}
\tau=\frac{1}{\sqrt{2 E} \sin \beta \cosh \left(T_{1} \cos \beta\right)}+O(\alpha) \\
\tau_{1}=-\frac{1}{\tau \cos \beta}\left(\frac{1}{\cos \beta}-\Delta T \operatorname{coth}\left(T_{1} \cos \beta\right)\right)+O\left(\alpha^{3}\right) .
\end{gathered}
$$

Note that $\tau_{1} \sim O(\alpha), \tau \sim O(1 / \alpha)$. Then, the real part of Eq. (55) implies that

$$
\cosh \left(T_{1} \cos \beta\right)=\frac{1}{\sin \beta}\left[1+\alpha \cot \beta \cos \tau e^{\Delta T}\right]+O\left(\alpha^{2}\right) .
$$

While deriving this formula we imposed $T_{1}<0$, which follows from the requirement that in the limit $\alpha \rightarrow 0$ Eq. (31) should be recovered; besides, we assumed $e^{\Delta T} \sim O(1)$. Substituting Eq. (58) into Eq. (56) and the imaginary part of Eq. (55), we obtain the final set of equations,

$$
\begin{aligned}
& 1-\tau \sqrt{2 E}=\alpha \cot \beta \cos \tau e^{\Delta T}+O\left(\alpha^{2}\right), \\
& (1+\Delta T) e^{-\Delta T}=\alpha \cot \beta \tau \sin \tau+O(\alpha) .
\end{aligned}
$$

These two nonlinear equations, still, cannot be solved explicitly. Nevertheless, one can obtain a pretty accurate idea about the structure of their solutions.

Before proceeding to the analysis of the above equations, let us derive a convenient expression for the suppression exponent $F_{0}(E) \equiv F(E, N=0)$. Note that on general grounds one expects to obtain an expression of the form

$$
F_{0}(E)=E\left[f_{\beta}(0)+O(\alpha)\right]
$$

where $f_{\beta}(0)$ is given by Eq. (33). We are interested in the $O(\alpha)$ correction in this expression, so, one must be careful to keep track of the subleading terms during the derivation.

Making use of the equations of motion, one obtains for the incomplete action (16) of the system,

$$
2 \operatorname{Im} \widetilde{S}=\operatorname{Im} p_{0}^{\prime}=\sqrt{2 E} \sin \beta \operatorname{Im}\left(\cos \phi_{1}\right) .
$$

Substitution of Eqs. (56)-(58) into this formula yields

$$
\begin{aligned}
2 \operatorname{Im} \widetilde{S}= & 2 E\left\{-1-\Delta T-\alpha \cot \beta \cos \tau e^{\Delta T}\right. \\
& \left.\times\left(1+\frac{1}{\cos ^{2} \beta}+2 \Delta T\right)+O\left(\alpha^{2}\right)\right\} .
\end{aligned}
$$

For the parameter $T$ one has [see Eqs. (14)]

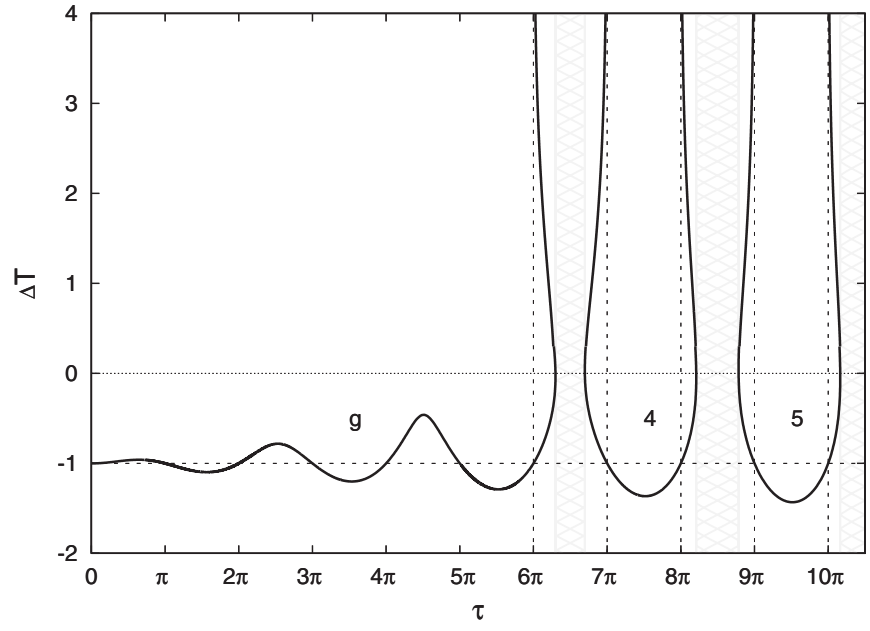

FIG. 7. Curves representing solutions to Eq. (59b); $\beta=\pi / 3, \alpha$ $=\pi / 30$.

$$
\begin{aligned}
T & =-\frac{2 \operatorname{Im} x_{0}}{p_{0}}=-\frac{2 \operatorname{Im}\left[x\left(t_{0}\right)-p_{0} t_{0}\right]}{p_{0}} \\
& =2\left(T_{1}-\Delta T\right)+\sqrt{\frac{2}{E}} \sin \alpha \operatorname{Im} y^{\prime}\left(t_{0}\right),
\end{aligned}
$$

where in the last equality we used Eqs. (34) and $x^{\prime}\left(t_{0}\right)=0$. The quantity $\operatorname{Im} y^{\prime}\left(t_{0}\right)$ is evaluated by using Eqs. (52b), (53), and (58); one finds

$$
\operatorname{Im} y^{\prime}\left(t_{0}\right)=-\sqrt{2 E}\left[\cot \beta \cos \tau e^{\Delta T}+O(\alpha)\right] .
$$

Substituting everything into formula (15), we obtain

$$
F_{0}(E)=E\left[f_{\beta}(0)-4 \alpha \cot \beta \cos \tau \Delta T e^{\Delta T}+O\left(\alpha^{2}\right)\right] .
$$

This expression implies that determination of the $O(\alpha)$ correction to the suppression exponent involves finding $\tau, \Delta T$ with $O(1)$ accuracy. This is precisely the level of accuracy of Eqs. (59). Below we will also need the following formulas, which can be easily obtained by using $T=-\frac{d F}{d E}$ and Eq. (60),

$$
\begin{aligned}
& \frac{d F_{0}}{d E}=f_{\beta}(0)+2(\Delta T+1)+O(\alpha), \\
& \frac{d}{d E}\left(\frac{F_{0}}{E}\right)=\frac{2[\Delta T+1+O(\alpha)]}{E} .
\end{aligned}
$$

Note that, though the suppression exponent differs from that in the one-turn case only by $O(\alpha)$ correction, its derivative is modified in the zero order in $\alpha$.

Now, we are ready to analyze Eqs. (59). One begins by solving Eq. (59b) graphically, see Fig. 7. The important property of this equation is as follows. One notices that the left-hand side of Eq. (59b) is always smaller than 1, the maximum being achieved at $\Delta T=0$. Therefore, the solutions to this equation are confined to the bands

$$
\tau \sin \tau<\frac{\tan \beta}{\alpha} .
$$

This corresponds to 

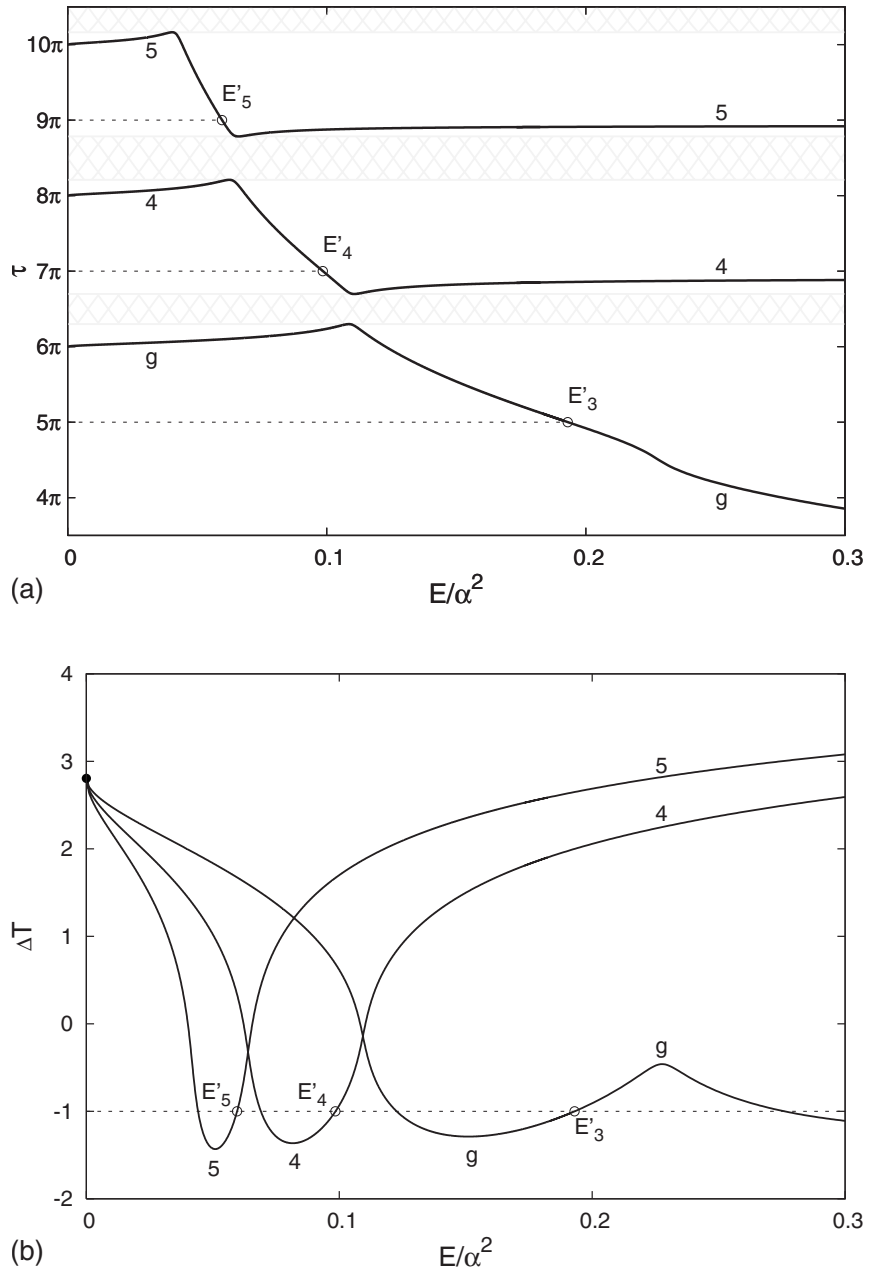

FIG. 8. Several first branches of solutions to Eqs. (59): "global" branch ("g") and two "local" branches ("4", "5"); $\beta=\pi / 3, \alpha$ $=\pi / 30$.

$$
\begin{gathered}
\tau \in\left[0 ; 2 \pi\left(n_{1}-1\right)+\delta \tau_{n_{1}}\right] \\
\text { or } \tau \in\left[2 \pi n-\pi-\delta \tau_{n} ; 2 \pi n+\delta \tau_{n}\right], \quad n \geq n_{1}
\end{gathered}
$$

where

$$
\begin{gathered}
\delta \tau_{n}=\arcsin \left(\frac{\tan \beta}{2 \pi \alpha(n-1 / 2)}\right)+O(\alpha), \\
n_{1}=\left[\frac{\tan \beta}{2 \pi \alpha}+\frac{1}{2}\right]+1,
\end{gathered}
$$

with [.] in the last formula standing for the integer part. The forbidden bands, where $\tau \sin \tau>\tan \beta / \alpha$, are marked in Fig. 7 by shading. The property (64) introduces a topological classification of the solutions $\tau, \Delta T$ to Eqs. (59). Namely, these solutions fall into a set of continuous branches: the local branches $\tau_{n}(E), \Delta T_{n}(E)$ living inside the strips $\tau$ $\in\left[2 \pi n-\pi-\delta \tau_{n} ; 2 \pi n+\delta \tau_{n}\right], n \geq n_{1}$, and the global branch $\tau_{g}(E), \Delta T_{g}(E)$ inhabiting the very first band $\tau \in\left[0 ; 2 \pi\left(n_{1}\right.\right.$ $\left.-1)+\delta \tau_{n_{1}}\right]$. As follows from the definition of $\tau$, the topologi- cal number $n$ counts the number of $y^{\prime}$ oscillations during the evolution in the intermediate region.

Let us consider the global branch. From Eqs. (59) one has

$$
\begin{gathered}
\tau_{g} \rightarrow 2 \pi\left(n_{1}-1\right)+O(\alpha \ln \alpha), \quad \Delta T_{g} \rightarrow \ln (\tan \beta / \alpha), \\
\text { as } E \rightarrow 0, \\
\tau_{g} \rightarrow 0, \quad \Delta T_{g} \rightarrow-1, \quad \text { as } E \rightarrow+\infty
\end{gathered}
$$

By inspection of Fig. 7 one can work out the qualitative behavior of the functions $\tau_{g}(E), \Delta T_{g}(E)$. Alternatively, these functions can be found numerically. They are plotted in Fig. 8 for the case $\beta=\pi / 3, \alpha=\pi / 30$ (the curves marked with " $g$ "). One observes that at high enough energies the function $\Delta T_{g}(E)$ exhibits oscillations around the line $\Delta T=-1$. According to formula (63) this means that the function $F_{0}(E) / E$ is nonmonotonic, it attains local minima at the points

$$
E_{n}^{\prime}=\frac{1}{8 \pi^{2}(n-1 / 2)^{2}}\left[1+2 \alpha e^{-1} \cot \beta+O\left(\alpha^{2}\right)\right]
$$

Moreover, if

$$
n \geq n_{0}^{\prime} \equiv\left[\frac{\tan \beta}{4 \pi \alpha} f_{\beta}(0) \exp \left(1+\frac{f_{\beta}(0)}{2}\right)+\frac{1}{2}\right]+1
$$

there exist $E_{n}^{o}=E_{n}^{\prime}[1+O(\alpha)]$, such that $\Delta T\left(E_{n}^{o}\right)=-1$ $-f_{\beta}(0) / 2$. Then, according to Eq. (62) the points $E_{n}^{o}$ are the optimal energies corresponding to the local minima of the suppression exponent $F_{0}(E)$.

At low energies the function $\Delta T_{g}(E)$ ceases to oscillate and becomes large and positive. According to Eq. (62) this means that the suppression exponent $F_{0, g}(E)$ of the global solution becomes negative at low energies, ${ }^{12}$ see Fig. 9. This is a clear signal that the global solution becomes unphysical at these energies and its contribution to the reflection probability should be discarded: negative suppression exponent contradicts the unitarity requirement, ${ }^{13} \mathcal{P}<1$. One is forced to conclude that at low energies reflection is described by the local solutions. Let us study them in detail.

For the $n$th branch one obtains

$$
\begin{gathered}
\tau_{n} \rightarrow 2 \pi n+O(\alpha \ln \alpha), \quad \Delta T_{n} \rightarrow \ln (\tan \beta / \alpha), \quad E \rightarrow 0, \\
\tau_{n} \rightarrow 2 \pi n-\pi, \quad \Delta T_{g} \rightarrow+\infty, \quad E \rightarrow+\infty
\end{gathered}
$$

From Fig. 7 one learns that the $n$th solution passes through the points

\footnotetext{
${ }^{12}$ It is worth mentioning that Eqs. (59) and the expression (61) for the suppression exponent become inapplicable at large $\Delta T$ : the assumption $e^{\Delta T} \sim O(1)$ which was used in the derivation of these equations is violated. Nevertheless, by analyzing the full Eqs. (54) and (55) one can show that $d F_{0, g} / d E=-T_{g}$ is large and positive at $E \rightarrow 0$. This is sufficient for concluding that $F_{0, g}(E)$ is negative in the low-energy domain.

${ }^{13}$ Another indication that the global solution is unphysical at small $E$ is that the function $\tau_{g}(E)$ is bounded from above. Indeed, $\tau$ is the time interval the particle spends in the intermediate part of the waveguide, one expects it to tend to infinity as $E \rightarrow 0$ for a physically relevant solution.
} 


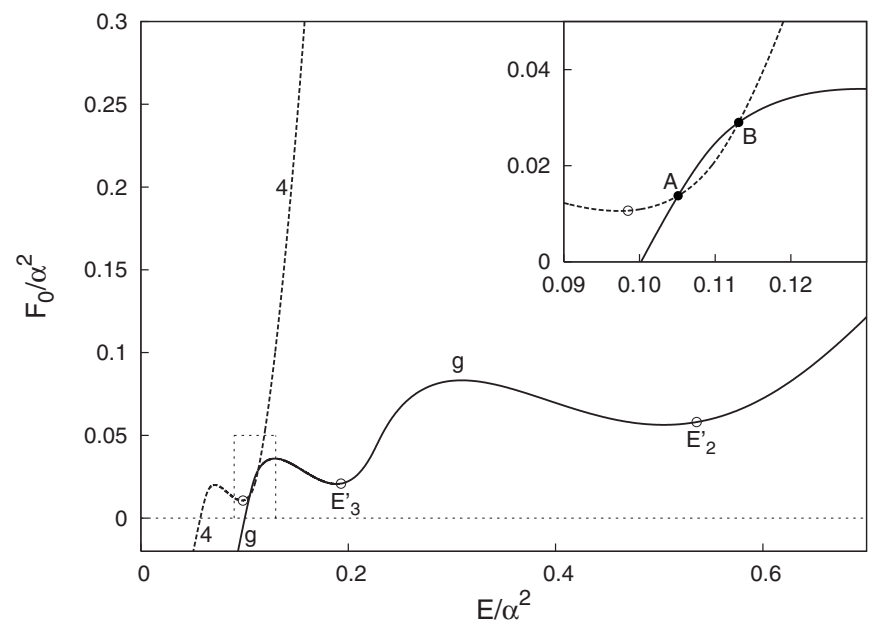

FIG. 9. The suppression exponent $F_{0}(E)$ for the global and first local $(n=4)$ branches; $\beta=\pi / 3, \alpha=\pi / 30$. The vicinity of intersection of the graphs is enlarged in the upper right corner.

$$
\Delta T_{n}=-1, \quad \tau=2 \pi n \quad \text { or } \tau=2 \pi n-\pi
$$

Thus, each curve $\Delta T_{n}(E)$ has one sharp dip, its minimum is smaller than -1 , see Fig. 8. As in the case with the global branch, the points (68) represent the extrema of the functions $F_{0, n}(E) / E$; the positions of the local minima are again given by Eq. (66).

Making use of Eq. (61), we find that the suppressions $F_{0, n}(E)$ of the local branches are large and positive at high energies. Hence, these solutions give subdominant contributions to the reflection probability at such $E$ as compared to the global solution. As energy decreases, $F_{0, n}(E)$ also decreases, then makes one oscillation and drops to negative values at small $E$. The latter property means that each local branch becomes unphysical at small enough energies. The suppression exponent of the first local branch (corresponding to $n=4$ in the case $\beta=\pi / 3, \alpha=\pi / 30$ ) is presented in Fig. 9 .

An alert reader may have already guessed that we have met here the typical Stokes phenomenon [21]. In fact, the Stokes phenomenon is specific to the situations where some integral [e.g., the path integral (7) in our case] is evaluated by the saddle-point method. Essentially, it means the following: as one gradually changes the parameters of the integral in question, a given saddle point may become noncontributing after the values of these parameters cross a certain curve drawn in the parameter space, the Stokes line. Since the result of the computation should be continuous, this phenomenon occurs only for subdominant saddle points (saddlepoint trajectories in our case). Unfortunately, apart from several heuristic conjectures $[21,12]$, sometimes rather suggestive [13], there is presently no general method of dealing with the Stokes phenomenon in the semiclassical calculations. However, in the situation encountered above it suffices to use the simplest logic lying at the heart of all other approaches. ${ }^{14}$

When gathering the final result for the suppression exponent, we follow two guidelines. First, it is clear that, as energy decreases, each branch becomes unphysical before $F_{0, n}(E)$ crosses zero. On the other hand, at high energies one should pick up the branch corresponding to the smallest value of the suppression exponent. Looking at Fig. 9, one notes that the curves $F_{0, g}(E), F_{0,4}(E)$ have two intersections, $A$ and $B$. At $E>E_{B}$ one chooses the global branch. In the region $E_{A}<E<E_{B}$ we switch to the first local branch, because in this region $F_{0,4}(E)<F_{0, g}(E)$. Naively, at $E=E_{A}$ one should jump back to the global branch; however, in order to preserve unitarity at small energies, we suppose that somewhere in between the points $B$ and $A$ the global branch becomes noncontributing, so that one should stay at the local branch at $E<E_{A}$. Similarly, the adjacent local branches have two intersections; as the energy decreases, we switch from $n$th branch to $n+1$ at the first intersection, and stay there until the intersection with the $n+2$ branch. Overall, one obtains the graph for the suppression exponent plotted in Fig. 10. The suppression exponent oscillates between two linear envelopes, $F=E\left[f_{\beta}(0) \pm 4 e^{-1} \alpha \cot \beta\right]$; oscillations pile up in the region of low energies. The reflection process is optimal in the vicinities of the minima of the function $F_{0}(E)$.

\section{DISCUSSION}

By considering a class of two-dimensional waveguide models, we have demonstrated explicitly that the probability of overbarrier reflection can be a nonmonotonic function of energy. The origin of the effect lies in the classical dynamics: the parameters of the complex trajectory describing overbarrier reflection change quasiperiodically as the energy is decreased. This results in the oscillatory behavior of the suppression exponent. Reflection occurs with exponentially larger probability in the vicinities of optimal energies (local minima of the suppression exponent) while being highly suppressed in between.

Our results are obtained for a fairly specific class of waveguides, namely, the ones with very sharp turns. However, the qualitative features observed in this paper should be valid for quite general waveguide models: a classical particle with high energy feels any large-scale turn of the waveguide as a sharp one $;{ }^{15}$ if two turns are separated by a long interval of free motion, one arrives to the model (35). We remark that the phenomenon of optimal tunneling has been observed also in numerical investigation of a smooth waveguide, see Ref. [14].

The branch structure of solutions observed in the region of small energies is interesting from the mathematical point

\footnotetext{
${ }^{14}$ The simplification in the present case is related to the fact that we concentrate on the dominant semiclassical contribution, leaving aside the subdominant ones.

${ }^{15}$ More precisely, one should compare the width $b$ of the turn to the quantity $\frac{2 \pi}{\omega} \frac{p_{0}}{m}$, where $p_{0}$ is the translatory momentum of the particle and $\omega$ stands for the frequency of transverse oscillations; if $b \ll \frac{2 \pi p_{0}}{\omega m}$, one is in the class of models with sharp turns.
} 


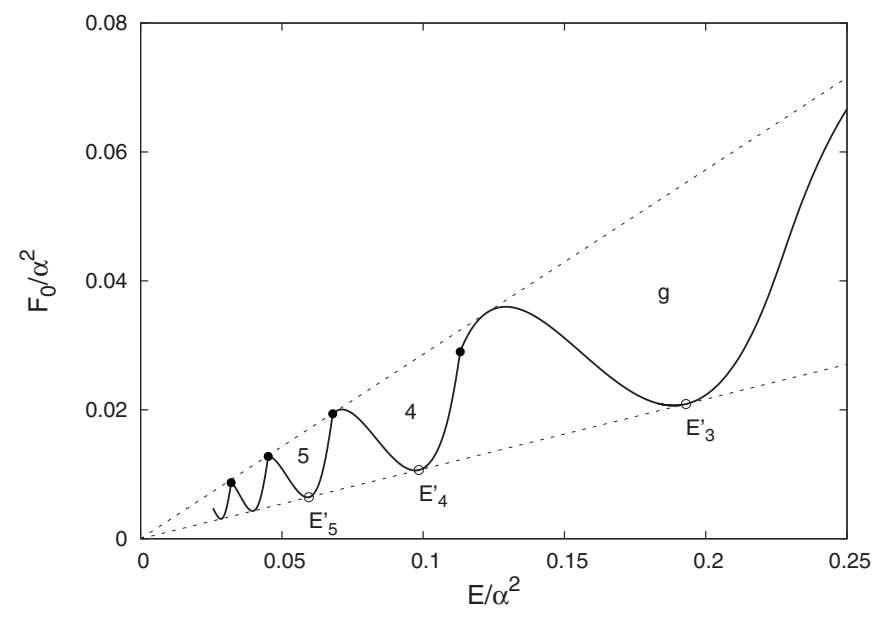

FIG. 10. The final result for the suppression exponent $F_{0}(E)$ in the region of small energies; $\beta=\pi / 3, \alpha=\pi / 30$. The points where different branches merge are shown with thick black dots.

of view. We have shown that there exists an infinite sequence of complex trajectories marked by the topological number $n$. Each branch produces physically consistent result for the suppression exponent in some energy interval; outside of this interval the $n$th branch would correspond either to highly suppressed transitions (high energies) or to violation of unitarity (low energies). We collected the final graph for the suppression exponent basing on the empirical considerations, which hardly may be acknowledged as satisfactory. Our study clearly shows that the method of complex trajectories should be equipped with a convenient rule to pick up the physical trajectory among the discrete set of solutions to the boundary value problem (9), (10), (12) (in other words, the method to deal with the Stokes phenomenon). Presently, such a rule is absent.

We note that the described physical phenomenon of optimal tunneling is present independently of the way the branches of solutions are glued together. The result at relatively high energies is given by the global branch, which displays a large number of local minima if $n_{0}^{\prime}>n_{1}$, see Eqs. (67) and (65). This is the case for the illustrative example considered throughout this paper, see Fig. 9.

As a final remark, we point out some open issues. We have calculated the suppression exponent of reflection using the sharp-turn approximation. It would be instructive to extend our analysis by finding corrections due to the finite turn widths. The motivation is twofold. First, the analysis performed in the Appendix implies the existence of a rich variety of distinctive semiclassical solutions contributing almost equally into the reflection probability. This feature might be a manifestation of chaos [7] which is present in our system but hidden by the sharp-turn approximation. (Note that chaos is inherent in a very similar waveguide model with smooth potential, see Ref. [14].) Clearly, the structure of solutions in the vicinities of the turns is worth further investigation.

Second, it was proposed recently in Refs. $[9,11]$ that the process of dynamical tunneling in quantum systems with multiple degrees of freedom (including field theoretical models, see Refs. [19]) can proceed differently from the ordinary case of one-dimensional tunneling. Namely, classically unstable state can be created during the process; this state decays subsequently into the final asymptotic region. The analysis performed in the present paper naturally conforms with this tunneling mechanism: all our complex trajectories are matched with the unstable orbit living at the turn. Still, the sharp-turn approximation does not allow to distinguish between the truly unstable trajectories staying at the turn forever and those which reflect from the turn in a finite time. To decide whether the tunneling mechanism of Refs. $[9,11]$ is indeed realized in our model, one needs to go beyond the sharp-turn approximation. Then, the candidate for the "mediator" unstable state is the "excited sphaleron," the solution considered in the Appendix. Presumably, in our model one can answer analytically to the question of whether or not the excited sphaleron acts as an intermediate state of the tunneling process. This study is quite beyond the scope of the present paper and we leave it for future investigations.

\section{ACKNOWLEDGMENTS}

We are indebted to F.L. Bezrukov and V.A. Rubakov for the encouraging interest and helpful suggestions. This work is supported in part by the Russian Foundation for Basic Research, Grant No. 05-02-17363-a; Grants of the President of Russian Federation No. NS-7293.2006.2 (government Contract No. 02.445.11.7370), No. MK-2563.2006.2 (D.L.), No. MK-2205.2005.2 (S.S.); Grants of the Russian Science Support Foundation (D.L. and S.S.); a grant from the "Dynasty" foundation (awarded by the Scientific board of ICFPM) (A.P.) and INTAS Grant No. YS 03-55-2362 (D.L.). D.L. is grateful to Universite Libre de Bruxelles and EPFL (Lausanne) for hospitality during his visits.

\section{APPENDIX: CLASSICAL MOTION NEAR THE TURN}

In this Appendix, we analyze the motion of the particle near the sharp turn of the waveguide (20) at nonzero smoothening of the turn, see, e.g., Eq. (21). We suppose that in the small vicinity of the turn the function $w(\xi, \eta)$ can be represented in the form

$$
w(\xi, \eta)=\cos \beta[\eta-b v(\xi / b)],
$$

where $v(\psi)$ does not depend explicitly on $b$. Moreover, we consider the case when $v(\psi)$ has a maximum, ${ }^{16}$

$$
v^{\prime}\left(\psi_{0}\right)=0 .
$$

Due to the property (A2) one immediately obtains the exact periodic solution to the equations of motion (9), which we call excited sphaleron [9],

$$
\xi_{\mathrm{sp}}=b \psi_{0}, \quad \eta_{\mathrm{sp}}=A_{\eta} \sin \left(t \cos \beta+\varphi_{\eta}\right)+b v\left(\psi_{0}\right) .
$$

We are going to show that this solution is unstable: a small perturbation above it grows with time and the particle flies away to either end of the waveguide. In particular, there are

\footnotetext{
${ }^{16}$ For the smoothening (21), the properties (A1) and (A2) hold with $v(\psi)=\frac{\psi \tan \beta}{1+e^{\psi}}, \psi_{0} \approx 1.28$.
} 
solutions that describe the decay of the sphaleron to $\xi \rightarrow-\infty$ both at $t \rightarrow \pm \infty$. Clearly, such solutions correspond to reflections from the turn.

In the vicinity of the sphaleron the trajectory of the particle can be represented in the form

$$
\xi=b \psi(t), \quad \eta=\eta_{\mathrm{sp}}(t)+b \rho(t),
$$

where $\psi, \rho \sim O(1)$. Writing down the classical equations of motion (9) in the leading order in $b$, one obtains

$$
\begin{gathered}
\frac{d^{2} \psi}{d s^{2}}=\frac{4}{b} A_{\eta} \sin (2 s) v^{\prime}(\psi), \\
\frac{d^{2} \rho}{d s^{2}}+4 \rho=4\left[v(\psi)-v\left(\psi_{0}\right)\right],
\end{gathered}
$$

where $s=\left(t \cos \beta+\varphi_{\eta}\right) / 2$. It is worth noting that the righthand side of Eqs. (A5) and (A6) are of different order in $b$. We will see that due to this difference $\rho=0$ in the leading order in $b$.

Let us first consider the linear perturbations above the excited sphaleron,

$$
\psi=\psi_{0}+\delta \psi, \quad \delta \psi \ll 1 .
$$

Equation (A5) can be linearized with respect to $\delta \psi$ leading to the Mathieu equation

$$
\frac{d^{2}}{d s^{2}} \delta \psi+2 q \sin (2 s) \delta \psi=0,
$$

with canonical parameter $q=-2 v_{0}^{\prime \prime} A_{\eta} / b>0$. As $q$ $\sim O(1 / b) \gg 1$, one can apply the WKB formula,

$$
\delta \psi=\frac{A \cos W}{\sqrt{d W / d s}},
$$

where $|A| \ll 1$, and

$$
W=\sqrt{2 q} \int_{\pi / 4}^{s} d s^{\prime} \sqrt{\sin \left(2 s^{\prime}\right)} .
$$

Note that we have chosen the solution symmetric with respect to time reflections,

$$
\delta \psi(\pi / 2-s)=\delta \psi(s)
$$

At $s \in[0 ; \pi / 2]$ the exponent $W$ is real and the particle is stuck at $\psi \approx \psi_{0}$, oscillating around this point with high frequency $d W / d s \sim O\left(b^{-1 / 2}\right)$. At $s<0$ the solution (A7) grows exponentially, meaning that the particle flies away from the excited sphaleron,

$$
\delta \psi(s<0)=\frac{A \cos [W(0)-\pi / 4]}{\sqrt{|d W / d s|}} e^{|W(s)-W(0)|} .
$$

In what follows, we choose $A \cos [W(0)-\pi / 4]<0$, so that $\delta \psi<0$ at $s<0$. Let us denote by $s_{1}<0$ the point where $\delta \psi$ becomes formally equal to -1 ,

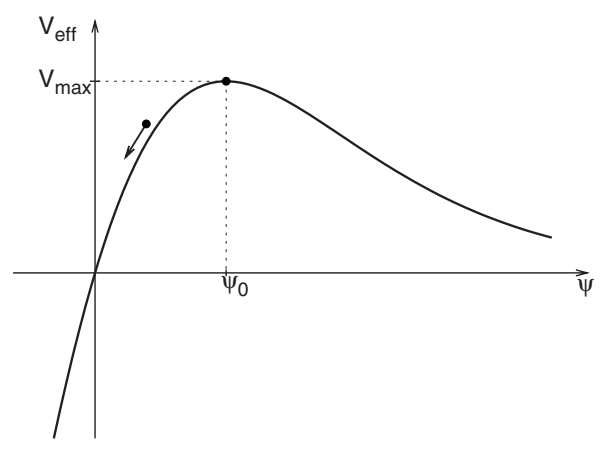

FIG. 11. The effective potential for Eq. (A10).

$$
\frac{A \cos [W(0)-\pi / 4]}{\sqrt{|d W / d s|}} e^{\left|W\left(s_{1}\right)-W(0)\right|}=-1 .
$$

In what follows we suppose that $s_{1} \sim O(1)$, hence, $A$ is exponentially small. Then, in the vicinity of this point, $\mid s$ $-s_{1} \mid \ll 1$, one has

$$
\begin{aligned}
\delta \psi & =-\exp \left[\sqrt{-2 q \sin \left(2 s_{1}\right)}\left(s_{1}-s\right)\right] \\
& =-\exp \left(\sqrt{4 v_{0}^{\prime \prime} A_{\eta} \sin \left(2 s_{1}\right)} \frac{\left(s_{1}-s\right)}{\sqrt{b}}\right) .
\end{aligned}
$$

We notice that $\delta \psi$ evolves from exponentially small values to $\delta \psi \sim O(1)$ during the characteristic time $\left|s-s_{1}\right| \sim O(\sqrt{b})$.

When $\delta \psi \sim O(1)$ the linear approximation breaks down and one has to solve the nonlinear equation (A5). Using $s$ $=s_{1}+O(\sqrt{b})$ one writes

$$
\frac{d^{2} \psi}{d s^{2}}=\frac{4}{b} A_{\eta} \sin \left(2 s_{1}\right) v^{\prime}(\psi)
$$

This equation permits us to draw a useful analogy with the one-dimensional particle moving in the effective potential $V_{e f f}(\psi)=-4 b^{-1} A_{\eta} \sin \left(2 s_{1}\right) v(\psi)$ (see Fig. 11). This auxiliary particle starts in the region near the maximum of the potential at $\left(s-s_{1}\right) / \sqrt{b} \rightarrow+\infty$ with energy $E \approx V_{\max }$ and rolls down toward $\psi \rightarrow-\infty$ at $\left(s-s_{1}\right) / \sqrt{b} \rightarrow-\infty$. In this limit $v(\psi)$ $\rightarrow \psi \tan \beta$ and the solution takes the form

$$
\psi=C_{1}+C_{2}\left(s-s_{1}\right)+2 b^{-1} A_{\eta} \sin \left(2 s_{1}\right) \tan \beta\left(s-s_{1}\right)^{2} .
$$

Note that the coefficients $C_{1}, C_{2}$ here are not independent: they are determined by the parameter $s_{1}$ through matching of the solution with Eq. (A9) at $\left(s-s_{1}\right) / \sqrt{b} \rightarrow+\infty$. We do not need their explicit form, however.

Let us argue that the function $\rho$ remains small during the whole evolution of the particle in the vicinity of the sphaleron. Indeed, in the linear regime one has $\delta \psi \ll 1$ and the right-hand side of Eq. (A6) is small. So, $\rho$ does not become excited. On the other hand, the nonlinear evolution of $\psi$ proceeds in a short time interval $\Delta s=O(\sqrt{b})$; so, again, $\rho$ is suppressed by some power of $b$.

The trajectory (A4) found in the vicinity of the sphaleron should be matched at 


$$
1 \gg\left|s-s_{1}\right| \gg \sqrt{b}
$$

with the free solution in the asymptotic region $\xi<0$, see Eqs. (26). It is straightforward to check that matching can be performed up to the second order in $\left(t-t_{1}\right)$, which is consistent with our approximations. In this way one determines the free asymptotic solution which, up to corrections of order $O(b)$, coincides with the sinusoid coming from $\xi \rightarrow-\infty$ at $t \rightarrow-\infty$ and touching the line $\xi=0$ at $t=t_{1}$.

Now we recall that, by construction, the obtained solution is symmetric with respect to time reflections,

$$
\xi(s)=\xi(\pi / 2-s), \quad \eta(s)=\eta(\pi / 2-s) .
$$

This means that it satisfies $\xi \rightarrow-\infty$ at $t \rightarrow \pm \infty$. This solution describes reflection of the particle from the turn.

The reasoning presented in this Appendix puts considerations of the main body of this paper on firm ground: we have found the "smoothened" solutions which reflect classically from the turn, and in the limit $b \rightarrow 0$ coincide with the free solutions of Sec. III touching the line $\xi=0$.

It is worth mentioning that, apart from the reflected solution we have found, in the vicinity of any trajectory touching the line $\xi=0$ there exists a rich variety of qualitatively dif- ferent motions. First of all, one may successfully search for solutions which are odd with respect to time reflections [Eq. (A8) with minus sign]. Such solutions, though close to the reflected ones at $t<0$, describe transmissions of the particle through the sharp turn into the asymptotic region $\xi \rightarrow+\infty$. Relaxing the time reflection symmetry, one can find solutions leaving the vicinity of the turn at any point $\eta<0$, which is different, in general, from the starting point $\eta=\eta\left(s_{1}\right)$. Yet other types of solutions are obtained in the case when the amplitude $A$ of $\delta \psi$ oscillations at $s \in[0 ; \pi / 2]$ is so small that $\delta \psi$ does not reach the values of order one during the time period $s \in[-\pi / 2 ; 0]$. If the particle is still in the vicinity of the point $\psi_{0}$ at $s=-\pi / 2$, it remains for sure in this vicinity at $s \in[-\pi ;-\pi / 2]$, because the right-hand side of Eq. (A5) is positive again. In this way one obtains solutions, which spend two, three, etc. sphaleron periods at $\psi \approx \psi_{0}$ before escaping into the asymptotic regions $\psi \rightarrow \pm \infty$. In the leading order in $b$ all these solutions correspond to the identical initial state, and (in the case of classically forbidden transitions) to the same value of the suppression exponent. However, an accurate study of the dynamics in the vicinity of the sphaleron is generically required to obtain the correct value of the suppression exponent in the case $b \sim 1$, cf. Ref. [14].
[1] V. A. Kuzmin, V. A. Rubakov, and M. E. Shaposhnikov, Phys. Lett. 155B, 36 (1985).

[2] W. Miller and T. George, J. Chem. Phys. 56, 5668 (1972); 57, 2458 (1972); W. H. Miller, Adv. Chem. Phys. 25, 69 (1974).

[3] A. M. Perelomov, V. S. Popov, and M. V. Terent'ev, Zh. Eksp. Teor. Fiz. 51, 309 (1966); V. S. Popov, V. Kuznetsov, and A. M. Perelomov, ibid. 53, 331 (1967).

[4] M. Davis and E. Heller, J. Chem. Phys. 75, 246 (1981).

[5] M. Wilkinson, Physica D 21, 341 (1986); S. Takada and H. Nakamura, J. Chem. Phys. 100, 98 (1994); S. Takada, P. N. Walker, and M. Wilkinson, Phys. Rev. A 52, 3546 (1995); S. Takada, J. Chem. Phys. 104, 3742 (1996).

[6] W. Miller, J. Phys. Chem. A 105, 2942 (2001).

[7] O. Bohigas, D. Boose, R. Egydio de Carvalho, and V. Marvulle, Nucl. Phys. A 560, 197 (1993); S. Tomsovic and D. Ullmo, Phys. Rev. E 50, 145 (1994); A. Shudo and K. S. Ikeda, Phys. Rev. Lett. 74, 682 (1995); Physica D 115, 234 (1998); E. Doron and S. D. Frischat, Phys. Rev. Lett. 75, 3661 (1995); S. D. Frischat and E. Doron, Phys. Rev. E 57, 1421 (1998); S. C. Creagh and N. D. Whelan, Phys. Rev. Lett. 77, 4975 (1996).

[8] G. F. Bonini, A. G. Cohen, C. Rebbi, and V. A. Rubakov, Phys. Rev. D 60, 076004 (1999).

[9] F. Bezrukov and D. Levkov, Zh. Eksp. Teor. Fiz. 125, 938 (2004); [J. Exp. Theor. Phys. 98, 820 (2004)]; e-print arXiv:quant-ph/0301022.

[10] C. S. Drew, S. C. Creagh, and R. H. Tew, Phys. Rev. A 72,
062501 (2005).

[11] K. Takahashi and K. S. Ikeda, Europhys. Lett. 71, 2, 193 (2005); Phys. Rev. Lett. 97, 240403 (2006).

[12] S. Adachi, Ann. Phys. (N.Y.) 195, 45 (1989).

[13] A. Shudo and K. S. Ikeda, Phys. Rev. Lett. 76, 4151 (1996).

[14] D. G. Levkov, A. G. Panin, and S. M. Sibiryakov, e-print arXiv:nlin.cd/0701063, Phys. Rev. E (to be published).

[15] A. S. Ioselevich and E. I. Rashba, Zh. Eksp. Teor. Fiz. 91, 1917 (1986); [Sov. Phys. JETP 64, 1137 (1986).]

[16] M. B. Voloshin, Phys. Rev. D 49, 2014 (1994).

[17] D. G. Levkov and S. M. Sibiryakov, Phys. Rev. D 71, 025001 (2005); Pis'ma Zh. Eksp. Teor. Fiz. 81, 60 (2005); [JETP Lett. 81, 53 (2005).]

[18] M. P. Mattis, Phys. Rep. 214, 159 (1992); P. G. Tinyakov, Int. J. Mod. Phys. A 8, 1823 (1993); V. A. Rubakov and M. E. Shaposhnikov, Usp. Fiz. Nauk 166, 493 (1996); [Phys. Usp. 39, 461 (1996).]

[19] V. A. Rubakov, D. T. Son, and P. G. Tinyakov, Phys. Lett. B 287, 342 (1992); A. N. Kuznetsov and P. G. Tinyakov, ibid. 406, 76 (1997); F. Bezrukov, D. Levkov, C. Rebbi, V. A. Rubakov, and P. Tinyakov, Phys. Rev. D 68, 036005 (2003)

[20] A. Ringwald, Nucl. Phys. B 330, 1 (1990); O. Espinosa, ibid. 343, 310 (1990).

[21] P. V. Elyutin and V. D. Krivchenkov, Nonrelativistic Quantum Mechanics in Problems (Fizmatlit, Moscow, 2001); M. V. Berry and K. E. Mount, Rep. Prog. Phys. 35, 315 (1972). 